\title{
Análisis de la calidad del dato de un instrumento de observación del clima motivacional: conductas verbales y proxémicas de monitores de mantenimiento físico.
}

\section{Data quality analysis of a motivational climate observation instrument: verbal and proxemic behaviours of physical maintenance monitors.}

\section{Análise da qualidade dos dados de um instrumento de observação do clima motivacional: comportamentos verbais e proxémicos dos monitores de manutenção física.}

\author{
Crespillo-Jurado, M. ${ }^{1}$, Anguera, M.T. ${ }^{2}$, Reigal, R.E. ${ }^{3}$, Hernández-Mendo, A. ${ }^{4}$ \\ 1,3,4 Universidad de Málaga, Málaga, España $;^{2}$ Universidad de Barcelona, Barcelona, España.
}

\begin{abstract}
RESUMEN
En este estudio se ha diseñado un instrumento para la observación del clima motivacional a través del análisis de la conducta verbal y proxémica en monitores que imparten la actividad de mantenimiento físico. Se trata de un instrumento que permite diferenciar el clima motivacional favorecido por el entrenador, así como la distribución de los participantes y la proxémica. El instrumento combina formato de campo, sistemas de categorías exhaustivos y mutuamente excluyentes (E/ME), y rating scales. El sistema está formado por 10 criterios y 44 categorías en total, y de cada una de ellas se elaboró una rating scale (a excepción de tres criterios en que no era posible). El nombre del instrumento es IAFD (Interacción en Actividad Física y Deporte). Se realizó la grabación, visualización y análisis de siete sesiones de mantenimiento físico en el Polideportivo Municipal de la ciudad de Ronda, analizando las conductas verbales y proxémicas de dos monitores. Se ha realizado un análisis de la calidad del dato y un análisis de generalizabilidad, obteniéndose resultados satisfactorios en cuanto a la fiabilidad, validez y precisión del instrumento, invarianza entre los dos monitores, así como de la capacidad de generalización.
\end{abstract}

Palabras clave: Metodología observacional, rating scales, generalizabilidad, invarianza, calidad del dato. 


\title{
Análisis de la calidad del dato de un instrumento de observación del clima motivacional
}

\begin{abstract}
In this study, an instrument has been designed for the motivational climate observation through the verbal and proxemic behaviour analysis in monitors who impart physical maintenance activity. It is an instrument that allows to differentiate the motivational climate favoured by the trainer, as well as the distribution of the participants and the proxemic. The instrument combines field format, comprehensive and mutually exclusive category systems (E/ME), and rating scales. It consists of 10 criteria and 44 categories in total, each of which (except for those criteria that were not possible) has rating scales. The name of the instrument is IAFD (Interaction in Physical Activity and Sport). The recording, visualization, and analysis of seven physical maintenance sessions in the Municipal Sports Centre of the city of Ronda was carried out, analysing the verbal and proxemic behaviours of two monitors. An analysis of data quality and an analysis of generalizability was carried out, obtaining satisfactory results in terms of the reliability, validity and precision of the instrument, invariance between the two monitors, as well as the capacity of generalization.
\end{abstract}

Keywords: Observational methodology, rating scales, generalizability, invariance, data quality.

\section{RESUMO}

Neste estudo, foi concebido um instrumento para a observação do clima motivacional através da análise do comportamentos verbais e proxémicos em monitores que realizam atividade de manutenção física. É um instrumento que permite diferenciar o clima motivacional favorecido pelo formador, assim como a distribuição dos participantes e a proxemia. $\mathrm{O}$ instrumento combina formato de campo, sistemas de categoria abrangentes e mutuamente exclusivos (E/ME), e escalas de classificação. O sistema consiste em 10 critérios e 44 categorias no total, cada uma das quais (com excepção dos critérios que eram sistemas de categorias) tem escalas de classificação. O nome do instrumento é IAFD (Interação em Atividade Física e Esporte). Foi feita a gravação, visualização e análise de sete sessões de manutenção física Polideportivo Municipal da cidade de Ronda, analisando os comportamentos verbais e proxêmicos de dois monitores. Foi realizada uma análise da qualidade dos dados e uma análise de generalização, obtendo resultados satisfatórios em termos de confiabilidade, validade e precisão do instrumento, invariância entre os dois monitores, assim como a capacidade de generalização.

Palavras chave: Metodologia observacional, escalas de classificação, generalizabilidade, invariância, qualidade dos dados.

\section{INTRODUCCIÓN}

La interacción ha sido estudiada desde hace mucho tiempo por autores clásicos que diseñaron instrumentos de observación en el ámbito de los grupos o en relación de liderazgo (Bales, 1950; Bales y Cohen, 1979; Borgatta y Crowther, 1965; Mann, 1966;), así como se ha observado desde diferentes perspectivas, como son el ámbito de la psicología clínica (Collie y Lingiardi, 2009; Del Giacco et al., 2019), el ámbito académico (Hamre et al., 2013; Pedrosa et al., 2013; Tronchoni et al., 2018), áreas más en auge como la interacción en redes sociales (Azagra y González, 2015), o el ámbito deportivo (Lapresa et al., 2020; Mendes et al., 2013; Santos et al., 2014).

En el estudio de esta interacción la metodología observacional presenta un perfil, en cuanto a la gran flexibilidad como a la inmensa cantidad de objetivos diversos a los que permite dar respuesta, el cual posibilita obtener grandes beneficios (Anguera y Hernández Mendo, 2013). Además, esta metodología considera todas las etapas del método científico, permitiendo coincidir con los requisitos de objetividad y rigurosidad (Anguera y Hernández Mendo, 2014) lo cual ha dado lugar al desarrollo, mejora y creación de nuevas herramientas de observación.

En este sentido se han creado instrumentos como el Coach Behavioral Assessment (CBAS) de Smith et al. (1977) y el más reciente Sistema de Observación del Clima Motivacional Multidimensional (MMCOS), (Fabra et al., 2018; Smith et al., 2015). Estos instrumentos se centran en el estudio de los climas motivacionales favorecidos por el entrenador, aunque con diferencias notables en 


\section{Crespillo-Jurado, M., Anguera, M. T., Reigal, R. E., Hernández-Mendo, A.}

cuanto al objetivo de estudio o la distinción que tienen. Mientras que el CBAS (Smith et al., 1977) estudia el comportamiento de los entrenadores sin distinguir entre un clima orientado a la tarea o al ego (RodríguezPeláez et al., 2015), el MMCOS (Fabra et al., 2018) en cambio, sí distingue entre estos climas, y se basa en el modelo propuesto por Duda (2013) diferenciando dos tipos de climas favorecidos por el entrenador, "clima empowering" y "clima disempowering". Este planteamiento une los conceptos de: (1) la teoría de Metas de Logro (Achievement Goal Theory, AGT) de Ames (1992) y Nicholls (1989): orientación al ego y orientación a la tarea; (2) la Teoría de la Autodeterminación (Deci y Ryan, 1985, 2000), en concreto, la tercera de las miniteorías, la Teoría de las Orientaciones de Causalidad (COT, Causality Orientations Theory): orientación de autonomía y orientación de control; (3) el apoyo social (Duda, 2013).

La AGT propone que el clima motivacional que favorecen los entrenadores influye en como los deportistas perciben su competencia y definen el éxito (Ames, 1992; Duda y Balaguer, 2007; Nicholls, 1989), distinguiendo dos tipos de clima motivacional: implicado en la tarea e implicado en el ego. En el clima motivacional implicado en la tarea, el entrenador favorece el aprendizaje cooperativo, reconoce el esfuerzo y la mejora, así como valora la importancia del papel del propio jugador, en este caso la competencia es percibida y evaluada en función de criterios autorreferenciados (Duda y Balaguer, 2007) En el polo opuesto, el clima motivacional implicado en el ego, donde el entrenador favorece la rivalidad, hace distinciones en función de parámetros como la habilidad entre los jugadores, y si se cometen errores se aplica un castigo (Newton et al., 2000). La competencia y habilidad es percibida en función de criterios normativos, en la comparación con otros.

En cuanto a la Teoría de la Autodeterminación (SDT, Self-Determination Theory; Deci y Ryan, 1985, 2000) se pueden diferenciar principalmente dos estilos interpersonales diferentes desde la microteoría de la Evaluación Cognitiva (Cognitive Evaluation Theory, CET): (1) el estilo de Apoyo a la Autonomía, en este estilo el entrenador reconoce los intereses de los atletas, las perspectivas y preferencias, y anima a los atletas a tomar posesión de su participación (Deci y Ryan, 1987); (2) el estilo Controlador, en el cual el entrenador impone de una forma autoritaria y coercitiva una determinada forma de pensar o actuar a los jugadores (Bartholomew et al., 2010).

Considerando las cuestiones planteadas, Duda (2013) propone un modelo integrador y multidimensional del clima motivacional donde distingue dos climas motivacionales diferentes: (1) clima "empowering", en el cual el entrenador presenta un estilo de apoyo a la Autonomía, una implicación en la tarea y proporciona apoyo social; y (2) clima "disempowering", antagonista al anterior, en el cual el entrenador desarrolla un estilo controlador, implicación en el ego y se frustra el apoyo social.

En relación con el desarrollo del instrumento presentado en este trabajo, inicialmente se realizó una revisión teórica de los instrumentos ya existentes en la temática, y se consideraron como referentes la versión en castellano de Sistema de Observación del Clima Motivacional Multidimensional (MMCOS) (Fabra et al., 2018), el Coach Behavioral Assessment (CBAS) de Smith et al. (1977), y el Interaction Process Analysis (IPA) de Bales (1951). Asimismo, se tuvo también en cuenta la proxémica de Hall (1963) y el propio aprovechamiento del espacio en función de la agrupación de los participantes por parte del monitor.

Uno de los aspectos más innovadores añadido al instrumento, IAFD (Interacción en Actividad Física y Deporte), ha sido el desarrollo e inclusión de las ratings scales a cada categoría existente, con excepción de los criterios Silencio (8), Aprovechamiento del espacio (9) y Proxémica (10).

Estas escalas corresponden a un sistema dimensional de registro, que actualmente tienen un carácter residual debido al necesario requisito de ordenación del atributo o dimensión, que no siempre es posible ni fácil (Anguera y Hernández Mendo, 2013). Esta cuestión pretende ser estudiada, permitiendo una mayor visualización y futuro uso de las rating scales.

Los objetivos de este trabajo son: (1) Diseñar y construir el instrumento de observación que permita valorar las conductas verbales de los monitores, así como la proxémica y el aprovechamiento del espacio. (2) Llevar a cabo un análisis de la fiabilidad, validez y precisión del instrumento elaborado. (3) Realizar un análisis de Generalizabilidad, que permita determinar la fiabilidad de los observadores, analizar la bondad de las categorías y estimar el número mínimo de sesiones necesarias para generalizar con precisión (BlancoVillaseñor et al., 2014). (4) Poner en valor la herramienta de ratings scales. 


\section{Análisis de la calidad del dato de un instrumento de observación del clima motivacional}

\section{MATERIAL Y MÉTODOS}

Diseño

En este trabajo se utiliza metodología observacional (Anguera, 1979). El diseño observacional (Anguera et al., 2011) utilizado pertenece al cuadrante IV, siendo N/S/M: nomotético, ya que se observa a dos monitores independientes; de seguimiento de carácter inter-sesional, puesto que se observan diferentes sesiones e intra-sesional al tener un registro de las conductas a observar a lo largo de toda la sesión, y, por último, se trata de un diseño multidimensional debido a la existencia de los diferentes criterios.

\section{Participantes}

Los participantes han sido dos monitores (un hombre y una mujer) con edades de 48 y 45 años, e impartiendo la actividad desde hace 22 y 12 años respectivamente. Esta actividad de Mantenimiento Físico para adultos y personas mayores, con edades comprendidas entre los 45 y los 85 años, tiene como objetivo lograr un acondicionamiento físico general, trabajando elementos de coordinación, movilidad articular, tonificación y definición muscular localizadas, a través del uso de diferentes materiales (bandas elásticas, mancuernas, fitball, esterillas, pelotas, etc.). La actividad era impartida en el Polideportivo Municipal de la ciudad de Ronda, en dos pistas polideportivas cubiertas y con una duración por sesión de 50 minutos. Se han realizado un total de 6.418 registros procedentes de la observación de un total de siete sesiones de mantenimiento físico (cuatro de un monitor y tres de otro).

\section{Material}

Para la grabación de las sesiones de mantenimiento físico se utilizaron dos cámaras Sony Handycam HDRCX625B.CEN, colocadas sobre sendos trípodes Sony y en la misma posición en cada una de las sesiones. La conducta verbal de los monitores se grabó mediante un micrófono inalámbrico de solapa Sennheiser EW 100 G3.

Para el registro, codificación y análisis de calidad del dato se utilizó el programa informático HOISAN (Hernández-Mendo, López-López, et al.,
2012). Posteriormente para la realización del análisis de Generalizabilidad se empleó el programa informático SAGT v1.0 (Hernández-Mendo, RamosPérez, et al., 2012). El análisis de la invarianza se realizó en una hoja de cálculo Excel.

\section{Instrumentos}

El instrumento de observación adaptado y elaborado ad hoc, es una combinación de formato de campo, sistema de categorías y rating scales (tabla 1, ver Anexo). Se cumple la exhaustividad y mutua exclusividad (Anguera et al., 2007) en cada uno de los sistemas de categorías que se han construido a partir de los criterios del instrumento, y a partir de cada categoría se ha elaborado una rating scale (excepto en unas pocas excepciones en que no era posible por su naturaleza, en los criterios 8,9 y 10). El instrumento está formado por 10 criterios y 44 categorías en total. El nombre del nuevo instrumento es IAFD (Interacción en Actividad Física y Deporte). Mantiene la estructura del MMCOS (Fabra et al., 2018), salvo ciertos cambios, como el presentar los criterios: Silencio (S); Aprovechamiento del espacio (AE), que hace referencia a como el monitor distribuye a los participantes en ese momento; y Proxémica $(\mathrm{P})$, que valora la distancia a la que se encuentra el monitor de la mayoría de los participantes. Este instrumento, además, presenta la inclusión de ratings scales a cada una de las categorías, excepto en los criterios 8, 9 y 10. 


\section{Crespillo-Jurado, M., Anguera, M. T., Reigal, R. E., Hernández-Mendo, A.}

\section{Tabla 1}

Estructura del instrumento de observación: Criterios, categorías, códigos y ratings scales (ver Anexo)

\begin{tabular}{lll}
\hline \multicolumn{1}{c}{ Criterios } & \multicolumn{1}{c}{ Categorías } & Códigos \\
\hline 1. Apoyo a la & $\begin{array}{l}\text { 1.1 Reconoce } \\
\text { sentimientos y } \\
\text { Autonomía }\end{array}$ & RSP \\
& perspectivas &
\end{tabular}

Rating Scales

1: El monitor/a pregunta sobre cuestiones de sentimientos o puntos de vistas particulares de los participantes

2: El monitor/a tras una primera interacción con el participante hace algún gesto (no respuesta verbal)

3: El monitor/a tras la respuesta del participante vuelve a incidir con otra pregunta al respecto

4: El monitor/a tras la segunda respuesta del participante realiza un gesto (no respuesta verbal)

5: Tras la respuesta del participante el monitor responde dando una valoración personal

1.2 Proporciona POS 1: El monitor/a propone dos opciones.

opciones 2 : El monitor/a propone tres opciones.

significativas 3 : El monitor/a propone cuatro opciones o da a elegir dos.

4: El monitor/a propone cinco opciones.

5: El monitor/a propone que se haga lo que quiera.

1.3 Fomenta el FII 1: El monitor/a pregunta por los aspectos positivos de la actividad.

interés intrínseco

3: El monitor/a pregunta si ha gustado la actividad o no.

5: El monitor/a hace cambios relativos a lo que le responden los participantes.

1.4 Proporciona

PJ

justificación

1: El monitor/a proporciona una justificación que no aporta mucho simplemente es una cierta "aceptación", el tono no es de aspecto negativo.

2: El monitor/a proporcionaría una justificación de lo pedido desde su "opinión" considerando lo mejor para el participante.

3: El monitor/a proporciona una justificación más neutra, es decir, porque será mejor o peor en cada circunstancia, se quedaría en eso no daría más datos.

4: El monitor/a proporciona una breve explicación sobre porqué lo pide.

5: El monitor/a proporciona una explicación detallada de porqué lo pide.

1.5 Proporciona oportunidad para la aportación
1.6 Fomenta la toma de iniciativa activa

1.7 Fomenta la toma de iniciativa pasiva

\section{Controlador}

2.1 Utiliza recompensas extrínsecas
POP 1: El monitor/a permite que el participante diga algún comentario respecto a la actividad física que realiza.

2: El monitor/a permite que el participante haga una sugerencia respecto a modificaciones de la actividad.

3: El monitor/a permite que el participante de su opinión con respecto a la actividad que realiza.

4: El monitor/a permite que haga una acción que pueda ser una alternativa a la actividad de que realiza.

5: El monitor/a permite que el participante muestre la acción que quiere realizar al resto de la clase.

FTIA 1: El monitor/a presenta situaciones donde los participantes pueden tomar la iniciativa.

3: El monitor/a incita a que cada uno vaya tomando la iniciativa de forma explícita.

5: El monitor/a da carta libre y dice que cada uno a su ritmo dando total libertad.

FTIP 1: El monitor/a se encuentra pegado a los participantes, en encuentra dentro de la misma franja numérica en la figura 1.

2: El monitor/a se encuentra dentro de la franja numérica colindante en la figura 1.

3: El monitor/a se encuentra a dos franjas numéricas de los participantes en la figura

1.

4: El monitor/a se encuentra en la última franja numérica en la figura 1.

5: El monitor/a se encuentra en la línea más lejana y fuera del campo.

URE 1: El monitor/a emplea un gesto por la correcta realización de la tarea.

2: El monitor/a da una respuesta verbal por la realización correcta de la tarea.

3: El monitor/a ofrece una respuesta verbal acompañada de un gesto por la correcta realización de la tarea.

4: El monitor/a ofrece respuesta verbal y contacto por la correcta realización de la tarea. 


\section{Análisis de la calidad del dato de un instrumento de observación del clima motivacional}

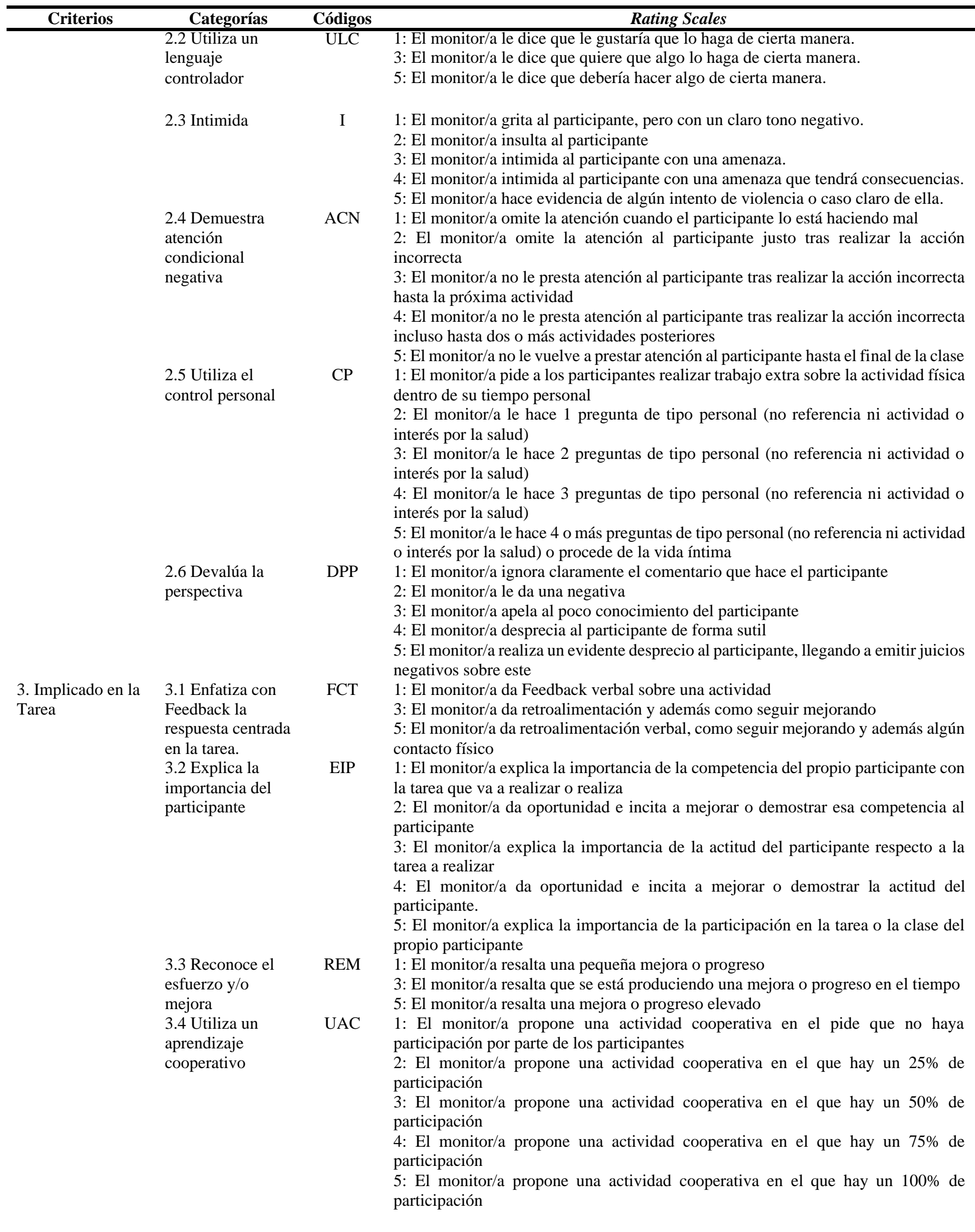




\section{Crespillo-Jurado, M., Anguera, M. T., Reigal, R. E., Hernández-Mendo, A.}

\begin{tabular}{ll}
\hline \multicolumn{1}{c}{ Criterios } & \multicolumn{1}{c}{ Categorías } \\
\hline $\begin{array}{l}\text { 4. Implicado en el } \\
\text { Ego }\end{array}$ & $\begin{array}{l}\text { 4.1 Penaliza los } \\
\text { errores }\end{array}$ \\
& \\
& \\
& 4.2 Reconoce \\
diferencias de \\
rendimiento y \\
habilidad \\
\\
\\
4.3 Fomenta la \\
rivalidad \\
intragrupo \\
5. Apoyo a la & 5.1 Se asegura la \\
inclusión de los \\
& participantes \\
\end{tabular}

Códigos

$\mathrm{P}$

$1: \mathrm{B}$

1: El Rating Scales

1: El monitor/a amonesta verbalmente al participante tras un error realizado.

2: El monitor/a saca una "tarjeta amarilla" es decir podríamos considerarlo más "grave"

3: El monitor/a saca una "tarjeta roja" no tiene porqué ser como en fútbol que signifique la expulsión del partido sino considerarlo más "grave"

4: El monitor/a expulsa fuera del juego a un participante tras cometer un error

5: El monitor/a pone un castigo al participante tras cometer un error

diferencias de

RIS 1: El monitor/a le enfatiza que lo hace peor que otro, una comparativa negativa

2: El monitor/a hace una comparación positiva, enfatizando el superior

3: El monitor/a enfatiza cierta igualdad que puede ser positiva o negativa

4: El monitor/a enfatiza que "tiene que hacerlo mejor que..."

5: El monitor/a enfatiza en superlativo

FR 1: El monitor/a dice en clase que es importante ser superior a los otros

3: El monitor/a en casos particulares dice cuanto es necesario para superar a otros

5: El monitor/a recalca sobre un caso concreto que ha superado a otro

API 1: El monitor/a le repite la información de nuevo mencionando o refiriendo a una persona

2: El monitor/a le repite la información de nuevo mencionando o refiriendo a dos personas

3: El monitor/a le repite la información de nuevo mencionando o refiriendo a tres o más personas

4: El monitor/a adapta un ejercicio para que puedan llevarlo a cabo

5: El monitor/a adapta una actividad para que todos puedan realizar la tarea

5.2 Permite conversaciones no

CNI 1: El monitor/a solo escucha a los participantes hablar sobre temas que no tienen que ver con la clase o actividad física que realizan instruccionales

5.4 Muestra interés y preocupación por los participantes

5.5 Está pendiente del participante

5.6 Bromea

5.7 Pide disculpas

PD 1: El monitor/a matiza algo previamente para que no se tome a mal por parte de los participantes

3: El monitor/a se disculpa en general delante de todos

5: El monitor/a pide disculpas a alguien personalmente

5.8 Da afecto

1: El monitor/a dice "Hola" a algún participante (o todos) cuando llegan.

2: El monitor/a dice "Hola" acompañada de otra fórmula como "buenas", "buenos días", “Qué tal?" (este último hay que diferenciarlo de que no se trata de un interés en sí por el participante sino como fórmula de cortesía.

3: El monitor/a hace algún gesto de afecto

4: El monitor/a ofrece algún recurso al participante

5: El monitor/a ofrece ayuda al participante
6. Frustración de
6.1 Excluye a
la Relación
participantes

EP 1: El monitor/a excluye no se dirige o evita el contacto con un participante

2: El monitor/a excluye de las explicaciones a algún participante

3: El monitor excluye sin motivo a algún participante de un ejercicio en particular

4: El monitor/a excluye sin motivos a varios participantes de algún ejercicio

5: El monitor/a excluye sin motivo a algún participante de una actividad completa 


\section{Análisis de la calidad del dato de un instrumento de observación del clima motivacional}

\begin{tabular}{|c|c|c|c|}
\hline Criterios & Categorías & Códigos & Rating Scales \\
\hline & $\begin{array}{l}6.2 \text { Delimita las } \\
\text { oportunidades de } \\
\text { interacción }\end{array}$ & $\overline{\mathrm{DOI}}$ & $\begin{array}{l}\text { 1: El monitor/a no continua una conversación que le propone alguno de los } \\
\text { participantes } \\
\text { 3: El monitor/a calla a algún participante o pequeño grupo de participantes que están } \\
\text { hablando }\end{array}$ \\
\hline & & & 5: El monitor/a manda a callar a todo el grupo \\
\hline & $\begin{array}{l}6.3 \text { Muestra una } \\
\text { falta de cuidado y } \\
\text { preocupación }\end{array}$ & MFC & $\begin{array}{l}\text { 1: El monitor/a omite la atención a algún participante concreto } \\
\text { 3: El monitor/a no se interesa si le pasa algo a algún participante que le pueda } \\
\text { repercutir en la propia clase }\end{array}$ \\
\hline & & & $\begin{array}{l}\text { 5: El monitor/a no se interesa si en el momento le pasa algo a algún participante } \\
\text { durante la propia clase }\end{array}$ \\
\hline & 6.4 Menosprecia & M & 1: El monitor/a realiza una falta de "consideración" leve \\
\hline & & & 3: El monitor/a hace una valoración negativa al participante \\
\hline \multirow{20}{*}{ 7. Estructurado } & & & 5: El monitor/a pone en evidencia la participante delante de los demás \\
\hline & 7.1 Proporciona & PIOA & 1: El monitor/a dice "cambio" y no da más detalle al respecto. \\
\hline & instrucciones y & & 2: El monitor/a se refiere a la parte del cuerpo \\
\hline & orientación antes & & 3: El monitor/a indica la parte del cuerpo, así como la dirección del movimiento \\
\hline & de la tarea & & $\begin{array}{l}\text { 4: El monitor/a amplia la información anterior con otro dato al respecto } \\
\text { 5: El monitor/a además de la ampliación realiza una demostración de lo que los } \\
\text { participantes deben realizar }\end{array}$ \\
\hline & 7.2 Proporciona & POD & 1: El monitor/a dice "cambio" y no da más detalle al respecto. \\
\hline & instrucciones y & & 2: El monitor/a se refiere a la parte del cuerpo \\
\hline & orientación & & 3: El monitor/a indica la parte del cuerpo, así como la dirección del movimiento \\
\hline & durante la tarea & & 4: El monitor/a amplia la información anterior con otro dato al respecto \\
\hline & & & $\begin{array}{l}\text { 5: El monitor/a además de la ampliación realiza una demostración de lo que los } \\
\text { participantes deben realizar }\end{array}$ \\
\hline & 7.3 Ofrece & OEA & 1: El monitor/a apela a una acción que se realizó en el pasado \\
\hline & $\begin{array}{l}\text { expectativas para } \\
\text { el aprendizaje }\end{array}$ & & $\begin{array}{l}\text { 2: El monitor/a apela a un conocimiento ya existente (la diferencia radica en que } \\
\text { antes teníamos la acción igual y ahora es el concepto ya aprendido) }\end{array}$ \\
\hline & & & 3: El monitor/a se refiere al presente objetivo \\
\hline & & & 4: El monitor/a apela a un futuro real \\
\hline & & & $\begin{array}{l}\text { 5: El monitor/a apela a un futuro hipotético o situaciones totalmente distinta a la } \\
\text { actividad, pero en las que puede servir }\end{array}$ \\
\hline & $\begin{array}{l}7.4 \mathrm{Da} \\
\text { sugerencias }\end{array}$ & DS & $\begin{array}{l}\text { 1: El monitor/a da una sugerencia para alcanzar una meta, del tipo "Podrías hacerlo } \\
\text { así...". }\end{array}$ \\
\hline & & & 2: El monitor/a al ver al participante realizarlo mal podría decirle cómo hacerlo \\
\hline & & & $\begin{array}{l}\text { 3: El monitor/a le presenta al participante una alternativa a lo que está haciendo para } \\
\text { conseguir el fin perseguido }\end{array}$ \\
\hline & & & $\begin{array}{l}\text { 4: El monitor/a le presenta una explicación detallada de la realización de esa solución } \\
\text { o alternativa propuesta al ejercicio }\end{array}$ \\
\hline & & & 5: El monitor/a le suma una demostración de la acción a realizar \\
\hline \multirow{5}{*}{$\begin{array}{l}\text { 8. Silencio } \\
9 . \\
\text { Aprovechamiento } \\
\text { del espacio }\end{array}$} & 8.1 Silencio & $\mathrm{S}$ & \\
\hline & $\begin{array}{l}\text { 9.1 Junta a todo el } \\
\text { grupo }\end{array}$ & JG & \\
\hline & $\begin{array}{l}\text { 9.2 Separa } \\
\text { individualmente }\end{array}$ & SI & \\
\hline & $\begin{array}{l}9.3 \text { Pone en } \\
\text { parejas }\end{array}$ & PP & \\
\hline & $\begin{array}{l}\text { 9.4 Pone en } \\
\text { grupos }\end{array}$ & PG & \\
\hline \multirow[t]{4}{*}{ 10. Proxémica } & $\begin{array}{l}\text { 10.1 Distancia } \\
\text { íntima }\end{array}$ & DI & \\
\hline & $\begin{array}{l}10.2 \text { Distancia } \\
\text { personal }\end{array}$ & DP & \\
\hline & $\begin{array}{l}\text { 10.3 Distancia } \\
\text { social }\end{array}$ & DS & \\
\hline & $\begin{array}{l}\text { 10.4 Distancia } \\
\text { pública }\end{array}$ & $\mathrm{DPu}$ & \\
\hline
\end{tabular}




\section{Crespillo-Jurado, M., Anguera, M. T., Reigal, R. E., Hernández-Mendo, A.}

\section{Figura 1}

División de las zonas del campo en la categoría "Fomenta la toma de iniciativa pasiva, FTIP"

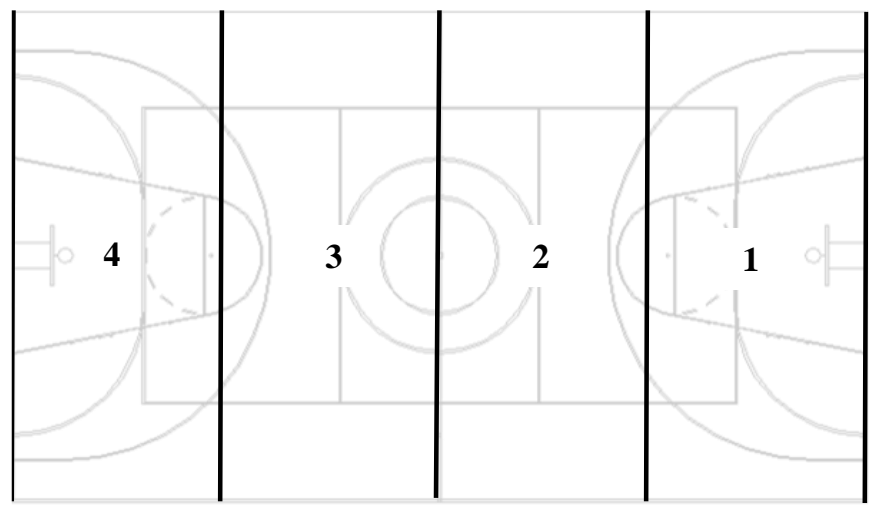

Procedimiento

El trabajo fue aprobado por el Comité de Ética de la Universidad de Málaga n 19-2015-H. Además, se siguieron las directrices de la Declaración de Helsinki (World Medical Association, 2013). Para iniciar la filmación de las sesiones se contó con la autorización del Ayuntamiento de Ronda, área de Deporte, Ocio y Tiempo Libre; así como se solicitó el Consentimiento Informado de monitores y participantes, que se realizaron de acuerdo con lo dispuesto en la Ley Orgánica 15/1999 de 13 de diciembre de Protección de Datos de Carácter Personal (LOPD). Este estudio cumple con las recomendaciones del Consejo General de Colegios de Psicólogos.

Inicialmente se filmaron 18 sesiones mediante dos cámaras de vídeo, colocadas en dos esquinas del campo para de esa forma tener la visión completa de todo el espacio en el que se llevaban a cabo las clases. Entre estas sesiones grabadas se eligieron tres sesiones del monitor 1 para realizar un análisis previo, fuera de muestreo, en esta selección se tuvo en cuenta que no hubiera dificultades tanto de imagen, por inobservabilidad, como de sonido. En esta fase previa se llevó a cabo un primer análisis de calidad del dato, valorándose la fiabilidad intra e inter. Tras este análisis preliminar se realizaron modificaciones en las categorías observadas y consensuadas entre observadores, así como se realizó un ajuste en las rating scales.

Posteriormente se filmaron otras 14 sesiones y de éstas se realizó el análisis final del instrumento. Las siete sesiones analizadas en este estudio se trataban de sesiones que distaban entre ellas el mismo espacio temporal (en este caso, un mes). Las sesiones se han nombrado como S1.1-S2.1-S3.1-S4.1-S1.2-S2.2-S3.2 (el número que acompaña a la letra " $S$ " corresponde con el número de sesión y el número que lo procede, el monitor que corresponde).

La filmación de un mayor número de sesiones a las analizadas se llevó a cabo para minimizar riesgos tales como que las grabaciones analizadas fueran de baja calidad (Anguera, 2003) o evitar la reactancia de los participantes observados (Anguera y HernándezMendo, 2014), ya que sabían que estaban siendo grabados; de esta forma al contar con tantas grabaciones los propios monitores y participantes de la actividad física se familiarizaron con la grabación y el observador que estaba presente.

El tipo de datos, de acuerdo con Bakeman (1978), son tipo IV, y, por tanto, concurrentes y tiempo-base. Esto significa que los datos están formados por cadenas de códigos co-ocurrentes (como máximo, uno por criterio o dimensión, al tratarse de un diseño multidimensional).

En este estudio la codificación, registro y análisis de calidad del dato se ha realizado con el programa HOISAN (Hernández-Mendo, LópezLópez, et al., 2012), el cual, con el uso de parámetros primarios y medidas derivadas o secundarias, lleva a cabo el cálculo de coeficientes de concordancia y acuerdo (Hernández-Mendo, López-López, et al., 2012). Se han obtenido los valores cuantitativos referentes a los coeficientes de correlación de Pearson, Spearman, la Tau-B de Kendall a nivel de frecuencias y en este caso al hacer uso de las rating scales también de intensidades, así como la estimación de la concordancia con el índice Kappa de Cohen.

Los observadores que han participado han sido dos, y se ha aplicado la concordancia consensuada (Anguera, 1990), estrategia de la metodología observacional que facilita el acuerdo entre observadores de forma cualitativa, realizándose la discusión sobre el código y alcanzando un consenso entre los observadores antes del registro (Castellano y Hernández-Mendo, 2000). Los observadores previamente observaron sesiones de entrenamiento fuera de muestreo para familiarizarles tanto con la actividad a observar como con el propio instrumento de observación. La formación que recibieron compartía la propuesta de Arana et al. (2016), a partir de Anguera (2003), y estaba compuesta por tres 


\section{Análisis de la calidad del dato de un instrumento de observación del clima motivacional}

bloques, una primera formación teórica en el propio instrumento de observación, una formación teóricopráctica del programa y el proceso de registro de las observaciones y por último la práctica autónoma del registro de una sesión.

Para el análisis, el observador 1 registró los datos de las sesiones S2.1 y S2.2 y posteriormente, transcurridos quince días, registró de nuevo estas mismas sesiones obteniéndose la concordancia intraobservador. El observador 2 una vez establecidos los acuerdos y la formación necesaria procedió al registro de ambas sesiones, obteniéndose la concordancia interobservador.

La calidad del dato también se ha evaluado desde la teoría de la Generalizabilidad (Cronbach et al., 1972), mediante el software informático SAGT (Hernández-Mendo, Ramos-Pérez, et al., 2012). Esta teoría permite analizar las diferentes fuentes de variación que pueden estar afectando a una medida o diseño de medida observacional, unificando los constructos de fiabilidad, validez y precisión (BlancoVillaseñor et al., 2014). Se distinguen cuatro etapas: (1) Definición de las facetas; (2) Análisis de varianza de las puntuaciones obtenidas sobre las facetas de estudio; (3) Cálculo de los componentes de error; (4) Optimización de los coeficientes de Generalizabilidad (Blanco-Villaseñor et al., 2014).

Por último, se ha evaluado la invarianza de medida, que se define con relación a un grupo o forma de una prueba, de tal modo que el significado formal y sustantivo de la medición es independiente respecto a ellos (Elosua, 2005). Formalmente se diría que la distribución de puntuaciones observadas depende sólo del espacio latente multidimensional (Mellenbergh, 1989; Meredith, 1993) del que aquéllas son indicadores.

\section{RESULTADOS}

En el análisis de la calidad del dato, se ha constatado que se tuviera constancia intersesional en cada una de las sesiones al cumplirse los siguientes requisitos: la sesión analizada ha sido del mismo grupo en cada monitor, se ha desarrollado siempre en el mismo lugar y con el mismo material.

\section{Concordancia entre observaciones}

Desde la vertiente cuantitativa de la concordancia, se han hallado para ambos monitores las correlaciones con los coeficientes Pearson, Spearman, Tau-b de Kendall, así como Kappa de Cohen.

\section{Tabla 2}

Coeficientes de correlación e índices Kappa de Cohen

\begin{tabular}{ccccc}
\hline & $\begin{array}{c}\text { Concordancia } \\
\text { Intra }\end{array}$ & \multicolumn{2}{c}{$\begin{array}{c}\text { Concordancia } \\
\text { Inter }\end{array}$} \\
\hline & $\begin{array}{c}\text { Monitor } \\
1\end{array}$ & $\begin{array}{c}\text { Monitor } \\
2\end{array}$ & $\begin{array}{c}\text { Monitor } \\
1\end{array}$ & $\begin{array}{c}\text { Monitor } \\
2\end{array}$ \\
\hline Pearson & .99 & .99 & .99 & .99 \\
Spearman & .99 & .99 & .99 & .99 \\
Tau b de & .99 & .99 & .99 & .99 \\
Kendall & & & & \\
(Intensidades) & & & & .99 \\
Tau b de & .99 & .99 & .99 & .99 \\
Kendall & & & & .93 \\
(Frecuencias) & & & & \\
Kappa de & .96 & .99 & .92 \\
Cohen & & & &
\end{tabular}

Los valores de los coeficientes de correlación presentados en la tabla 2 muestran unos resultados que permiten confirmar que la herramienta de observación ofrece un registro con alto grado de fiabilidad y precisión.

\section{Análisis de Invarianza}

En la invarianza de medida se ha empleado para la estimación de la invarianza, un contraste de hipótesis $\left(\mathrm{H}_{0}: \rho 1-\rho 2=0\right.$ y H1: $\left.\rho 1-\rho 2 \neq 0\right)$ se ha empleado la siguiente fórmula de $Z$ :

$$
\mathrm{Z}=\frac{(1 / 2) \ln \left[\left(1+r_{1}\right) /\left(1-r_{1}\right)\right]-(1 / 2) \ln \left[\left(1+r_{2}\right) /\left(1-r_{2}\right)\right]}{\sqrt{1 /\left(n_{1}-3\right)+1\left(n_{2}-3\right)}}
$$

donde:

ln $=$ logaritmo neperiano

$r_{1}=$ correlaciones del monitor 1

$r_{2}=$ correlaciones del monitor 2

$n_{1}=$ número de registros del monitor 1

$n_{2}=$ número de registros del monitor 2

En la tabla 3 aparecen los resultados obtenidos. En este caso los registros empleados han 


\section{Crespillo-Jurado, M., Anguera, M. T., Reigal, R. E., Hernández-Mendo, A.}

sido las sesiones analizadas para las concordancias, puesto que los coeficientes de correlación son los obtenidos en el propio análisis (tabla 2). Los resultados obtenidos muestran $Z$ con valores $\leq|1.96|$ por lo que las correlaciones no difieren de forma significativa para $\alpha=.05$.

\section{Tabla 3}

Contraste de hipótesis en muestras independientes respecto a los monitores

\begin{tabular}{|c|c|c|c|c|c|c|c|}
\hline \multicolumn{8}{|c|}{ Contraste de hipótesis } \\
\hline \multirow{4}{*}{$\begin{array}{c}\text { Pearson } \\
\text { Tau b } \\
\text { Kendall }\end{array}$} & $\begin{array}{l}\text { M1 } \\
\text { Intra }\end{array}$ & $\begin{array}{l}\text { M2 } \\
\text { Intra }\end{array}$ & & & & & \\
\hline & $\mathrm{r} 1$ & $\mathrm{r} 2$ & n1 & $\mathrm{n} 2$ & $\begin{array}{l}\text { Nume- } \\
\text { rador }\end{array}$ & $\begin{array}{l}\text { Deno- } \\
\text { mina- } \\
\text { dor }\end{array}$ & $\mathrm{Z}$ \\
\hline & .99 & .99 & 267 & 352 & $\overline{0}$ & .08 & 0 \\
\hline & .99 & .99 & 267 & 352 & 0 & .08 & 0 \\
\hline \multirow[t]{4}{*}{ Spearman } & .99 & .99 & 267 & 352 & 0 & .08 & 0 \\
\hline & M1 & M2 & & & & & \\
\hline & Inter & Inter & & & & & \\
\hline & $\mathrm{r} 1$ & $\mathrm{r} 2$ & $\mathrm{n} 1$ & n2 & $\begin{array}{l}\text { Nume- } \\
\text { rador }\end{array}$ & $\begin{array}{l}\text { Deno- } \\
\text { mina- } \\
\text { dor }\end{array}$ & $\mathrm{Z}$ \\
\hline Pearson & .99 & .99 & 270 & 354 & $\overline{0}$ & .08 & 0 \\
\hline Tau b & .99 & .99 & 270 & 354 & 0 & .08 & 0 \\
\hline Kendall & & & & & & & \\
\hline Spearman & .99 & .99 & 270 & 354 & 0 & .08 & 0 \\
\hline
\end{tabular}

\section{Análisis de Generalizabilidad}

Para determinar la fiabilidad entre los observadores (fiabilidad inter), se han usado los registros referentes a las sesiones (S2.1 y S2.2) analizadas por distinto observadores y se ha estimado un modelo de dos facetas, categorías y observadores $(\mathrm{C} / \mathrm{O})$, obteniéndose que el mayor porcentaje de la varianza está asociada a la faceta categorías $(99.65 \%)$ siendo nula para la faceta observadores, y de $0.35 \%$ para la faceta de interacción categorías/observadores. El análisis global de los coeficientes de generalizabilidad en este diseño, determina una fiabilidad de precisión en la capacidad de generalización de los resultados excelentes, obteniendo un índice de generalizabilidad relativo de .99 y un índice de generalizabilidad absoluto de .99 .
De la misma manera, para valorar la fiabilidad del mismo grupo de observadores (fiabilidad intra), en este caso se han usado los registros de las sesiones registradas por el mismo observador, se ha tomado el mismo diseño de dos facetas, categorías y observadores $(\mathrm{C} / \mathrm{O})$, y en este caso se ha obtenido que casi toda la variabilidad queda asociada a la faceta categorías (99.86\%) siendo nula para la faceta observadores, y de $0.14 \%$ para la faceta de interacción categorías/observadores. El análisis global de los coeficientes de generalizabilidad en este diseño, muestran un índice de generalizabilidad relativo de 1.00 y un índice de generalizabilidad absoluto de 1.00.

Respecto a la homogeneidad de las categorías, se ha optado de nuevo por un diseño de dos facetas, observadores y categorías $(\mathrm{O} / \mathrm{C})$, mediante el cual se pretende comprobar en qué grado las categorías propuestas diferencian las distintas acciones. Los coeficientes de generalizabilidad en este diseño son nulos (.00), por lo que se podría decir que la homogeneidad de las categorías resulta altamente diferenciadora.

Para estimar el número mínimo de sesiones necesarias para generalizar con precisión cualquier resultado se han empleado los registros de las sesiones S1.1-S2.1- S3.1-S4.1.Se ha utilizado un diseño de dos facetas, categorías y sesiones $(\mathrm{C} / \mathrm{S})$ obteniendo que gran parte de la variabilidad queda asociada a la faceta categorías $(67.89 \%)$ siendo nula para la faceta sesiones, y el resto para la faceta de interacción categorías/sesiones (32.11\%). El análisis global de los coeficientes de generalizabilidad en este diseño determina una fiabilidad de precisión en la generalización de los resultados con 5 sesiones de .90 (Maneiro et al., 2020) y de .95 con la observación de 9 sesiones (tabla 4 y Figura 2).

Además de los modelos de medida analizados para la valoración de la fiabilidad, la homogeneidad de las categorías y el número mínimo de sesiones necesarias para generalizar con precisión, se ha analizado el modelo [Criterios] [Categorías] / [Sesiones] [Monitores] obteniéndose con los dos monitores participantes y las siete sesiones (S1.1S2.1-S3.1-S4.1-S1.2-S2.2-S3.2), un coeficiente de generalizabilidad relativo de .84 y un coeficiente de generalizabilidad absoluto de .83 . 


\section{Análisis de la calidad del dato de un instrumento de observación del clima motivacional}

Tabla 4

Análisis de generalizabilidad para la optimización del diseño de medida

\begin{tabular}{|c|c|c|c|c|c|c|}
\hline $\begin{array}{l}\text { Nombre de } \\
\text { los valores }\end{array}$ & $\begin{array}{l}\text { Resu- } \\
\text { men }\end{array}$ & $\begin{array}{l}\text { Resu- } \\
\text { men } 2\end{array}$ & $\begin{array}{l}\text { Resu- } \\
\text { men } 3\end{array}$ & $\begin{array}{l}\text { Resu- } \\
\text { men } 4\end{array}$ & $\begin{array}{l}\text { Resu- } \\
\text { men } 5\end{array}$ & $\begin{array}{l}\text { Resu- } \\
\text { men } 6\end{array}$ \\
\hline Sesiones & $\begin{array}{l}(4 ; \\
\text { INF) }\end{array}$ & $\begin{array}{l}(5 ; \\
\text { INF) }\end{array}$ & $\begin{array}{l}(6 ; \\
\text { INF })\end{array}$ & $\begin{array}{l}(7 ; \\
\text { INF })\end{array}$ & $\begin{array}{l}(8 ; \\
\text { INF })\end{array}$ & $\begin{array}{c}(9 ; \\
\text { INF })\end{array}$ \\
\hline Categorías & $\begin{array}{l}(23 ; \\
\text { INF) }\end{array}$ & $\begin{array}{l}(23 ; \\
\text { INF) }\end{array}$ & $\begin{array}{l}(23 ; \\
\text { INF) }\end{array}$ & $\begin{array}{l}(23 ; \\
\text { INF) }\end{array}$ & $\begin{array}{l}(23 ; \\
\text { INF) }\end{array}$ & $\begin{array}{l}(23 ; \\
\text { INF) }\end{array}$ \\
\hline $\begin{array}{c}\text { Total } \\
\text { observacio- } \\
\text { nes }\end{array}$ & 92 & 115 & 138 & 161 & 184 & 207 \\
\hline $\begin{array}{l}\text { Coeficiente } \\
\text { G relativo }\end{array}$ & .89 & .91 & .93 & .94 & .94 & .95 \\
\hline $\begin{array}{l}\text { Coeficiente } \\
\text { G absoluto }\end{array}$ & .89 & .91 & .93 & .94 & .94 & .95 \\
\hline
\end{tabular}

\section{Figura 2}

Gráfica de generalización del Coeficiente $G$ absoluto en función del número de sesiones

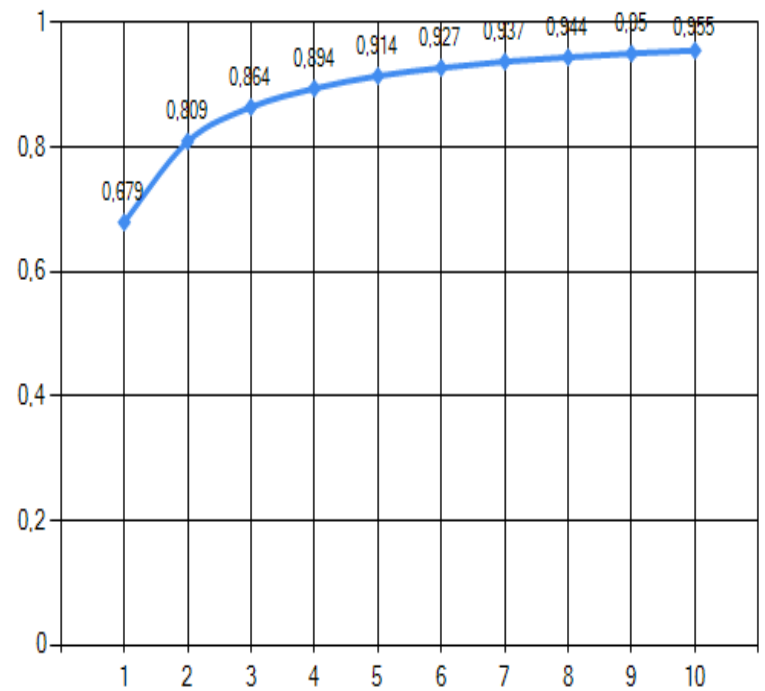

\section{DISCUSIÓN}

Los objetivos que presentaba este trabajo consistían, en primer lugar, en la construcción del propio instrumento en base a la teoría existente, incluyendo la dificultad añadida de la creación de las rating scales para cada una de las categorías presentadas con la salvedad de los criterios 8, 9 y 10 . Este primer proceso requirió de sucesivas adaptaciones hasta lograr el ajuste obtenido. Posteriormente se llevaron a cabo los análisis de la fiabilidad, validez y precisión de la herramienta de observación, que en base a los resultados obtenidos podemos afirmar que el instrumento dispone de estas características. Esto iría en la línea de estudios anteriores llevados a cabo con el Sistema de Observación del Clima Motivacional Multidimensional (Fabra et al., 2018; Smith et al., 2015).

La adaptación inicial se realizó inicialmente con los mismos participantes y fue analizada por dos observadores, los cuales recibieron una formación previa en el uso y manejo tanto del programa como del instrumento de observación. En este análisis se obtuvieron buenos resultados, aunque se produjo la mejora y adición en caso de necesidad de nuevas conductas. Posteriormente se realizó la filmación de nuevas sesiones y el consecuente análisis final con los mismos tanto participantes como observadores. Los resultados del análisis de calidad del dato reflejan una elevada fiabilidad, concordancia entre los observadores mediante el coeficiente Kappa de Cohen (1960) considerada como "muy buena o perfecta" (Kappa de Cohen > .80) a partir de los valores de referencia de Landis y Koch (1977).

Los resultados obtenidos en el análisis de invarianza entre los dos monitores demuestran que no hay diferencias entre ambos por lo que se produce invarianza del instrumento entre los monitores. En este aspecto, la inclusión de la invarianza tiene un carácter innovador ya que en la mayoría de las ocasiones se ha estudiado la invarianza factorial, valorando el grado en el que un instrumento mide el mismo constructo entre dos o más grupos (Pineda et al., 2018). Este estudio plantea esta invarianza desde el punto de vista del contraste de hipótesis a partir de las propias correlaciones, esta aplicación se ha empleado también en el trabajo de Quiñones et al. (2019).

Respecto a la generalizabilidad, determina una fiabilidad de precisión en la capacidad de generalización de los resultados excelentes, así como la homogeneidad de las categorías resulta altamente diferenciadora, además se ha realizado una optimización del modelo, que permite la obtención de referencias para futuros estudios. 


\section{Crespillo-Jurado, M., Anguera, M. T., Reigal, R. E., Hernández-Mendo, A.}

Lo realmente relevante e innovador de este estudio radica en el uso de las rating scales, que permiten realizar una primera muestra de las áreas o subáreas en las que se pretende medir el comportamiento producido, preparándose una serie de ejecuciones que están graduadas en cuanto a complejidad, dificultad, etc., indicando la asignación de puntuaciones (Anguera, 2001). Tas su análisis han mostrado que aunque tienen un uso residual debido a la necesidad de ordenación del atributo o dimensión, que no siempre es posible ni fácil (Anguera y Hernández Mendo, 2013), se presentan como una potencialidad, que mediante una profundización así como implantación de sistemas informáticos que permitan su explotación total pueden ofrecer grandes beneficios a la propia metodología, ofreciendo la posibilidad de obtener una información añadida y muy completa en los registros a realizar.

Como limitaciones en este estudio podría considerarse el número de monitores con los que ha contado el análisis de esta herramienta. Además, sería interesante la replicación en otros ámbitos diferentes a la actividad de mantenimiento físico, lo cual permitiría una mayor optimización del instrumento de observación. Debido a la naturaleza de la actividad en la cual se ha puesto a prueba este instrumento, encontramos que es una actividad con adultos y personas mayores, de carácter voluntaria y enfocada a la salud y bienestar, por tanto, hay criterios que conformarían el espectro más disempowering que carecen de frecuencia en el análisis realizado.

\section{APLICACIONES PRÁCTICAS}

Este estudio presenta un instrumento de observación que cumple los criterios de fiabilidad, validez y precisión. La aplicación práctica radica en el análisis de la interacción verbal y proxémica del monitor con los participantes de la actividad. Cabe mencionar que lo innovador y de gran relevancia metodológica en este estudio es el desarrollo de las rating scales como potencialidad. Aunque actualmente programas de observación como Hoisan trabaja actualmente con intensidades, este estudio pretende ser activador de la aplicación de este instrumento, lo cual posibilitará desarrollar y mejorar estas herramientas informáticas que permitan trabajar de manera óptima.

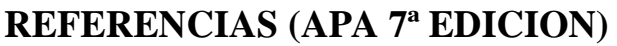

1. Ames, C. (1992). Classrooms: Goals, structures, and student motivation. Journal of Educational Psychology, 84(3), https://doi.org/10.1037/0022-0663.84.3.261

2. Anguera, M.T. (1979). Observational Typology. Quality \& Quantity. European-American Journal of Methodology, 13(6), 449-484. https://doi.org/10.1007/BF00222999

3. Anguera, M. T. (1990). Metodología observacional. En J. Arnau, M.T. Anguera y J. Gómez Benito. Metodología de la investigación en ciencias del comportamiento (pp.125236). Murcia: Universidad de Murcia.

4. Anguera, M. T. (2001). Cómo apresar las competencias del bebé mediante una aplicación de la metodología observacional. Contextos Educativos. Revista de Educación, (4), 13-34. https://doi.org/10.30827/publicaciones. v48i1.7331

5. Anguera, M. T. (2003). Observational Methods (General). En R. Fernández-Ballesteros (Ed.), Encyclopedia of Psychological Assessment, Vol. 2 (pp. 632-637). Londres: Sage. https://doi.org/ $10.4135 / 9780857025753$

6. Anguera, M. T., Blanco-Villaseñor, A., Hernández -Mendo, A., Losada, J. L. (2011). Diseños observacionales: ajuste y aplicación en psicología del deporte. Cuadernos de Psicología del Deporte, 11(2), 63-76.

7. Anguera, M. T., Magnusson, M., Jonsson, G. (2007). Instrumentos no estándar: planteamiento, desarrollo y posibilidades. Avances en Medición, 5(1), 63-82.

8. Anguera, M. T., Hernández-Mendo, A. (2013). La metodología observacional en el ámbito del deporte. E-balonmano.com: Revista de Ciencias del Deporte, 9(3), 135-160.

9. Anguera, M. T., Hernández-Mendo, A. (2014). Metodología observacional y psicología del deporte: Estado de la cuestión. Revista de Psicología del Deporte,23(1). 103-109.

10. Azagra, R., González, J. (2015). La interacción política en Twitter, el caso de@ ppopular y@ ahorapodemos durante la campaña para las 


\section{Análisis de la calidad del dato de un instrumento de observación del clima motivacional}

Elecciones Europeas de 2014. Ámbitos: Revista Internacional de Comunicación, 28,38-49.

11. Bakeman, R. (1978). Untangling streams of behavior: Sequential analysis of observation data. En G.P. Sackett (Ed). Observing behavior, Vol 2: Data collection and analysis methods (pp. 63-78). Baltimore: University of Park Press.

12. Bales, R. F. (1950). Interaction process analysis; a method for the study of small groups. Cambridge, Mass.: Addison-Wesley Press.

13. Bales, R. F., Cohen, S. P., Williamson, S. A. (1979). SYMLOG: A system for the multiple level observation of groups. Free Press.

14. Bartholomew, K. J., Ntoumanis, N., ThøgersenNtoumani, C. (2010). The controlling interpersonal style in a coaching context: Development and initial validation of a psychometric scale. Journal of Sport and Exercise Psychology, 32(2), 193-216. https://doi.org/10.1123/jsep.32.2.193

15. Blanco-Villaseñor, Á., Castellano, J., HernándezMendo, A., López-Sánchez, C. R., Usabiaga, O. (2014). Aplicación de la TG en el deporte para el estudio de la fiabilidad, validez y estimación de la muestra. Revista de Psicología del Deporte, 23(1), 131-137.

16. Borgatta, E.F., Crowther, B. (1965). A workbook for the study of social interaction processes. Chicago: Rand McNally.

17. Castellano, J., Hernández-Mendo, A. (2000). Generalizabilidad de las observaciones de la acción del juego en el fútbol. Psicothema, 12(Suplemento), 81-86.

18. Cohen, J. (1960). A coefficient of agreement for nominal scales. Educational and Psychological Measurement, $\quad 20(1), \quad 37-46$. https://doi.org/10.1177/001316446002000104

19. Colli, A., Lingiardi, V. (2009). The collaborative interactions scale: a new transcript-based method for the assessment of therapeutic alliance ruptures and resolutions in psychotherapy. Psychotherapy Research 19(6), 718-734. https://doi.org/ 10.1080/10503300903121098

20. Cronbach, L., Gleser, G., Nanda, H., Rajaratnam, N. (1972). The dependability of behavioral measurements: Theory of generalizability for scores and profiles. New York: John Wiley and Sons.

21. Deci, E. L., Ryan, R. M. (1985). Intrinsic motivation and self-determination in human behavior. New York, NY: Plenum.

22. Deci, E. L., Ryan, R. M. (2000). The" what" and" why" of goal pursuits: human needs and the selfdetermination of behavior. Psychological Inquiry, 11(4), 227-268. https://doi.org/10.1207/S15327965PLI1104_01

23. Deci, E.L., Ryan, R.M. (1987). The support of autonomy and the control of behavior. Journal of Personality and Social Psychology, 53, 10241037.https://doi.org/10.1037/00223514.53.6.1024

24. Del Giacco, L., Salcuni, S., Anguera, M. T. (2019). The communicative modes analysis system in psychotherapy from mixed methods framework: introducing a new observation system for classifying verbal and non-verbal communication. Frontiers in Psychology, 10:782. https://doi.org/10.3389/fpsyg.2019.00782

25. Duda, J. L. (2013). The conceptual and empirical foundations of Empowering Coaching ${ }^{\text {TM}}$ : Setting the stage for the PAPA project. International Journal of Sport and Exercise Psychology, 11(4), 311-318.

https://doi.org/10.1080/1612197X.2013.839414

26. Duda, J. L., Balaguer, I. (2007). Coach-created motivational climate. En S. Jowett y D. Lavallee (Eds.), Social Psychology in Sport (pp. 117-130). Champaign, IL: Human Kinetics. https://doi.org/10.5040/9781492595878.ch-009

27. Elosua, P. (2005). Evaluación progresiva de la invarianza factorial entre las versiones original y adaptada de una escala de autoconcepto. Psicothema, 17(2), 356-362.

28. Fabra, P., Balaguer, I., Tomás, I., Smith, N., Duda, J. L. (2018). Versión española del Sistema de Observación del Clima Motivacional Multidimensional (MMCOS): fiabilidad y evidencias de validez. Revista Psicología del Deporte, 27, 11-22.

29. Hall, E. T. (1963). A system for the notation of proxemic behaviour. American Anthropologist, 65(5), 1003-1026. 


\section{Crespillo-Jurado, M., Anguera, M. T., Reigal, R. E., Hernández-Mendo, A.}

30. Hamre, B.K., Pianta, R.C., Downer, J.

T., DeCoster, J., Mashburn, A. J., Jones, S. M., Brown, J. L., Capella, E., Atkins, M., Rivers, S.E., Brackett, M. A., Hamagami, A. (2013). Teaching through interactions: Testing a developmental framework of teacher effectiveness in over 4,000 classrooms. The Elementary School Journal, 113(4), 461-487.

https://doi.org/10.1086/669616

31. Hernández-Mendo, A., López-López, J., Castellano, J., Morales-Sánchez, V., Pastrana, J. L. (2012). Hoisan 1.2: Programa informático para uso en metodología observacional. Cuadernos de Psicología del Deporte, 12(1), 55-78. https://doi.org/10.4321/S1578842320120001000 06

32. Hernández-Mendo, A., Ramos-Pérez, F., Pastrana, J.L. (2012). SAGT: Programa informático para análisis de Teoría de la Generalizabilidad. SAFE CREATIVE Código: 1204191501059

33. Landis, R., Koch, G. (1977). The measurement of observer agreement for categorical data. Biometrics, 33(1),159-174. https://doi.org/10.2307/2529310

34. Lapresa, D., Pascual, J., Arana, J., Anguera, M. T. (2020). Sistema de observación para analizar la interacción en el juego de Boccia por equipos. Cuadernos de Psicología del Deporte, 20(1), 37-47. https://doi.org/10.6018/cpd.393821

35. Maneiro, R., Blanco-Villaseñor, Á., Amatria, M (2020) Analysis of the Variability of the Game Space in High Performance Football: Implementation of the Generalizability Theory. Frontiers in Psychology, 11:534. https://doi.org/ 10.3389/fpsyg.2020.00534

36. Mann, R. D. (1966). The development of the member-trainer relationship in self-analytic groups. Human Relations, 19(1), 85-115.

37. Mellenbergh, G. J. (1989). Item bias and item response theory. International Journal of Educational Research, 13, 127-143. https://doi.org/10.1016/0883-0355(89)90002-5

38. Mendes, S., Fernandes, J. J., Castañer, M., Camerino, O., de Sequeira, P. J. R. M., Dias, L. A., Da Costa, V.A., Alves, S. C. (2013). Sistema de observação da comunicação proxémica do instrutor de fitness (SOPROX-FITNESS): desenvolvimento, validação e estudo piloto. Revista Iberoamericana de Psicología del Ejercicio y el Deporte, 8(2), 281-299.

39. Meredith, W. (1993). Measurement invariance, factor analysis and factorial invariance. Psychometrika, 58, 525-543. https://doi.org/10.1007/BF02294825

40. Newton, M., Duda, J.L., Yin, Z.N. (2000). Examination of the psychometric properties of the Perceived Motivational Climate in Sport Questionnaire-2 in a sample of female athletes. Journal of Sports Sciences, 18, 275-290. https://doi.org/10.1080/026404100365018

41. Nicholls, J. G. (1989). The competitive ethos and democratic education. Cambridge, MA: Harvard University Press.

42. Pedrosa, I., Torres, N. H., Alegría, M. L., Cueto, E. G. (2013). Desarrollo del Protocolo de Observación de Interacción en el Aula: aplicación en un programa de niños con altas capacidades. Revista de Educación, 1, 293-312. https://doi.org/10.4438/1988-592X-RE-2013EXT-250

43. Quiñones, Y., Morillo-Baro, J. P., Reigal, R. E., Morales-Sánchez, V., Vázquez-Diz, J. A., Hernández-Mendo, A. (2019). El ataque posicional en balonmano: validación de un sistema de observación. Cuadernos de Psicología del Deporte, 19(3), 114-124. https://doi.org/10.6018/cpd.384091s

44. Rodríguez-Peláez, D., Garrido, P., Conde, C., Almagro, B. J. (2015). Adaptación y validación de la CBAS para la observación de climas motivacionales. Cuadernos de Psicología del Deporte, 15(3), 253-260. https://doi.org/10.4321/S157884232015000300030

45. Santos, S., Sarmento, H., Alves, J., Campaniço, J. (2014). Construcción de un instrumento para la observación y el análisis de las interacciones en el waterpolo. Revista de Psicología del Deporte, 23(1), 191-200.

46. Smith, N., Tessier, D., Tzioumakis, Y., Quested, E., Appleton, P., Sarrazin, P., Papaioannou, A., Duda, J. L. (2015). Development and validation of 


\section{Análisis de la calidad del dato de un instrumento de observación del clima motivacional}

the multidimensional motivational climate observation system. Journal of Sport and Exercise Psychology, 37(1),

4-22.

https://doi.org/10.1123/jsep.2014-0059

47. Smith, R.E., Smoll, F. L., Hunt, E. (1977). System for behavioral assessment of Athletic coaches. Research Quarterly, 48, 401-407. https://doi.org/10.1080/10671315.1977.10615438

48. Tronchoni, H., Izquierdo, C., Anguera M. T. (2018). Interacción participativa en las clases magistrales: fundamentación y construcción de un instrumento de observación. Publicaciones: Facultad de Educación y Humanidades del Campus de Melilla, 48(1), 81-108. https://doi.org/10.30827/publicaciones.

v48i1.7331
49. World Medical Association. (2013). World Medical Association Declaration of Helsinki: Ethical principles for medical research involving human subjects. Journal of the American Medical Association. 310, 2191-2194. https://doi.org/10.1001/jama.2013.281053 


\title{
Crespillo-Jurado, M., Anguera, M. T., Reigal, R. E., Hernández-Mendo, A.
}

\author{
Anexo
}

\begin{tabular}{|c|c|c|}
\hline Criterio & $\begin{array}{c}\text { Código: } \\
\text { Categoría }\end{array}$ & Definición/ Referencia \\
\hline $\begin{array}{l}1 . \\
\text { Apoyo a la } \\
\text { Autonomía }\end{array}$ & & $\begin{array}{l}\text { El entrenador reconoce los intereses de } \\
\text { los atletas, las perspectivas y } \\
\text { preferencias, y anima a los atletas a } \\
\text { tomar posesión de su participación }\end{array}$ \\
\hline & & $\begin{array}{l}\text { Deci, E.L., y Ryan, R.M. (1987). The } \\
\text { support of autonomy and the control of } \\
\text { behavior. Journal of Personality and } \\
\text { Social Psychology, 53(6), 1024-1037. } \\
\text { PubMed }\end{array}$ \\
\hline
\end{tabular}

1.1 RSP: $\quad$ El monitor demuestra que ha tenido en Reconoce cuenta los sentimientos y el punto de sentimientos y vista de los participantes.

perspectivas La relevancia radica en que el tema principal son los sentimientos de los participantes.

Smith, N., Tessier, D., Tzioumakis, MMCOS (Smith Y., Quested, E., Appleton, P., et al., 2015) Sarrazin, P., Papaioannou, A., y Duda, J. L. (2015). Development and validation of the multidimensional motivational climate observation system. Journal of Sport and Exercise Psychology, 37(1), 4-22.

1.2 POS:

Proporciona

opciones

significativas

El monitor presenta

varias opciones para trabajar un determinado objetivo, dando al participante la posibilidad de escoger.

Moreno, J. A., y Martínez, A. (2006). MMCOS (Smith Importancia de la Teoría de la et al., 2015) Autodeterminación en la práctica físico-deportiva: Fundamentos e implicaciones prácticas. Cuadernos de psicología del deporte, 6(2), 40-54.

1.3 FII: $\quad$ El monitor favorece o busca Fomenta el actividades que posean el atractivo de interés la novedad, el desafío, o valor estético. intrínseco $\quad$-Ryan, R., y Deci, E. L. (2000). La Teoría de la Autodeterminación y la Facilitación de la Motivación Intrínseca, el Desarrollo Social, y el MMCOS (Smith Bienestar. American et al., 2015) 1.4 PJ:

Proporciona justificación de tareas/peticiones /limitaciones
Psychologist, 55(1), 68-78.

El monitor explica o da una razón de por qué ha pedido que las cosas se hagan de una forma determinada.

MMCOS (Smith et al., 2015)
1: El monitor/a pregunta (o es receptivo/a) sobre cuestiones de sentimientos o puntos de vistas particulares de los participantes.

2: El monitor/a tras una primera interacción con el participante hace un movimiento de cabeza (no hay respuesta verbal).

3: El monitor/a tras la respuesta del participante vuelve a incidir con otra pregunta al respecto.

4: El monitor/a tras la segunda respuesta del participante realiza un movimiento de cabeza (no hay respuesta verbal). 5: Tras la respuesta del participante el monitor responde dando una valoración personal, por ejemplo, respondiendo "eso está muy bien", "lo veo perfecto por tu parte", etc.

1: El monitor/a propone dos opciones (ej.: "podéis elegir A o B").

2: El monitor/a propone tres opciones.

3: El monitor/a propone cuatro opciones o da a elegir dos (ej.: "podéis elegir entre $\mathrm{A}, \mathrm{B}$ o $\mathrm{C} /$ podéis elegir dos entre $\mathrm{A}$, B o C").

4: El monitor/a propone cinco opciones.

5: El monitor/a propone que se haga lo que quiera (ej.: "De brazos podéis hacer el que queráis").

1: El monitor/a pregunta por los aspectos positivos de la actividad, por ejemplo "espero que os haya parecido bien" o una pregunta directa tipo " $₫$ os ha parecido bien?".

3: El monitor/a pregunta si ha gustado la actividad o no por ejemplo "¿No os ha gustado? / ¿Os gusta?), "Espero que os haya gustado".

5: El monitor/a hace cambios relativos a lo que le responden los participantes por ejemplo "Como quiero que os divirtáis no vamos a hacer eso..."

1: El monitor/a proporciona una justificación que no aporta mucho simplemente es una cierta "aceptación" sería un "esto se hace así porque sí", el tono no es de aspecto negativo.

2: El monitor/a proporcionaría una justificación de lo pedido desde su "opinión" considerando lo mejor para el participante, por ejemplo, "realizad esto porque creo que es lo mejor para el músculo".

3: El monitor/a proporciona una justificación más neutra, es decir, porque será mejor o peor en cada circunstancia, se quedaría en eso no daría más datos. Por ejemplo "Esto es mejor así". 


\section{Análisis de la calidad del dato de un instrumento de observación del clima motivacional}

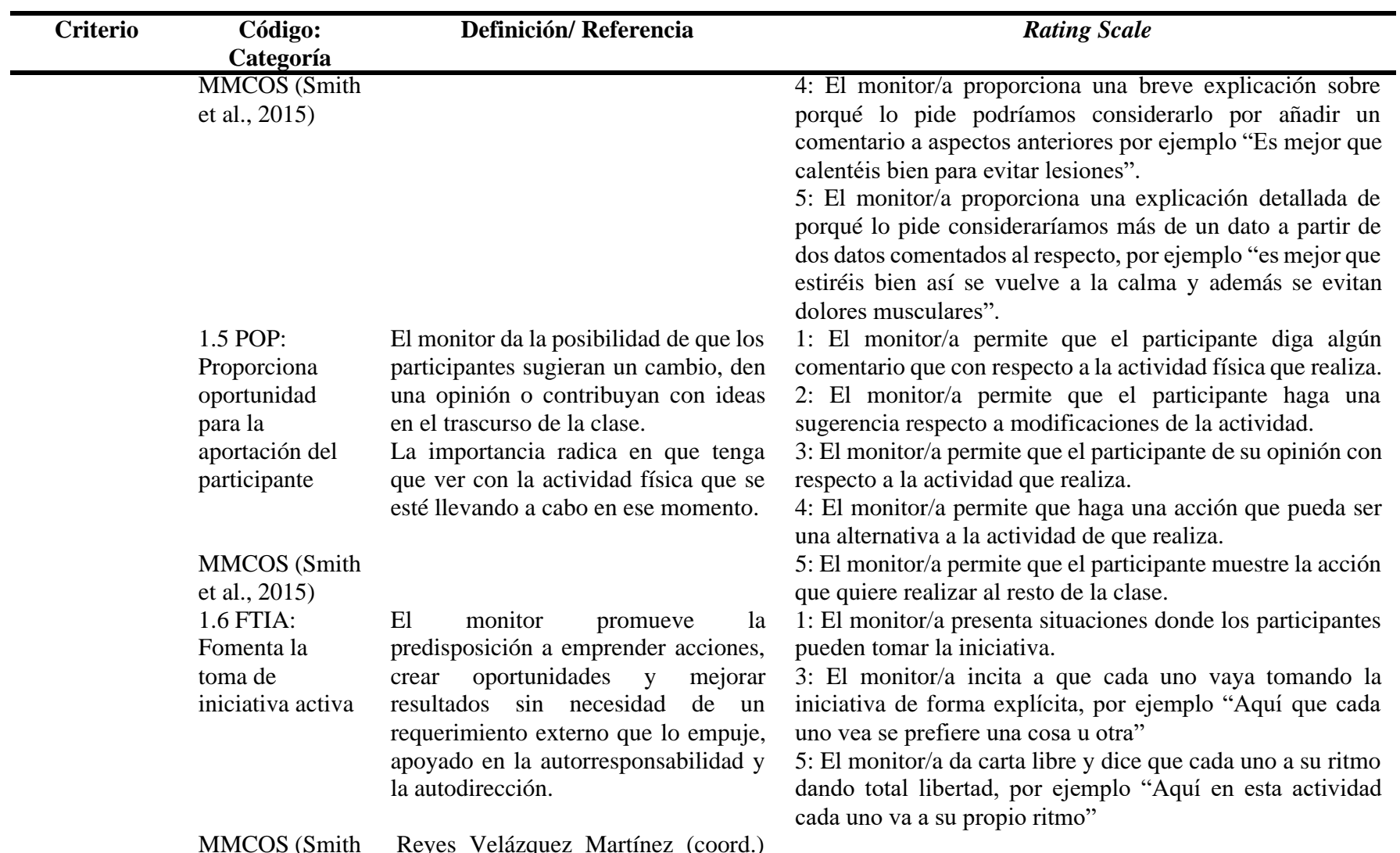

et al. 2015) (2009). Guía para la Formación en Competencias Profesionales para alumnos de la Universidad de Cádiz.

1.7 FTIP: Mantener la realización de las tareas Fomenta la sólo con la mera presencia del toma de monitor. No se observaría desde un iniciativa pasiva punto de vista autoritario sino desde el fenómeno de la "mera presencia" (Zajonc, 1965).

MMCOS (Smith et al., 2015)

2.

Controlador
Un estilo interpersonal de control se refleja cuando un entrenador coacciona el comportamiento de los atletas mediante el uso de estrategias de control y poder asertivo.

Smith, N., Tessier, D., Tzioumakis, Y., Quested, E., Appleton, P., Sarrazin, P., ... y Duda, J. L. (2015). Development and validation of the multidimensional motivational climate observation system. Journal of
1: El monitor/a se encuentra pegado a los participantes, en encuentra dentro de la misma franja numérica en la figura 1 (por ejemplo, podríamos considerarlo que están en la misma zona 1, o cualquiera de los números, pero en el mismo tramo) 2: El monitor/a se encuentra dentro de la franja numérica colindante en la figura 1 (por ejemplo, los participantes en la zona 1 y el monitor en la zona 2 )

3: El monitor/a se encuentra a dos franjas numéricas de los participantes en la figura 1 (por ejemplo, los participantes en la zona 1 y el entrenador en la zona 3 )

4: El monitor/a se encuentra en la última franja numérica en la figura 1 (por ejemplo, los participantes en la zona 1 y el entrenador en la zona 4)

5: El monitor/a se encuentra en la línea más lejana y fuera del campo

(Figura 1 al final del documento) 


\section{Crespillo-Jurado, M., Anguera, M. T., Reigal, R. E., Hernández-Mendo, A.}

\begin{tabular}{lll}
\hline Criterio & \multicolumn{1}{c}{$\begin{array}{c}\text { Código: } \\
\text { Categoría }\end{array}$} & \multicolumn{1}{c}{ Definición/ Referencia } \\
\hline & $\begin{array}{l}\text { Sport and Exercise Psychology, 37(1), } \\
4-22 .\end{array}$ \\
& $\begin{array}{l}\text { 2.1 URE: } \\
\text { Utiliza } \\
\text { recompensas } \\
\text { extrínsecas }\end{array}$ & $\begin{array}{l}\text { Hacer uso de recompensas de origen } \\
\text { externo tras la correcta realización de } \\
\text { la tarea. }\end{array}$ \\
& \\
& \\
MMCOS (Smith & \\
et al., 2015) & \\
2.2 ULC: & El monitor utiliza un lenguaje \\
Utiliza un & coercitivo para conseguir que los \\
lenguaje & jugadores cumplan \\
controlador & $\begin{array}{l}\text { sus demandas. No se trataría de un } \\
\text { consejo en tono amable. }\end{array}$ \\
MMCOS (Smith &
\end{tabular}

et al., 2015)

2.3 I: Se basa en la intimidación

MMCOS (Smith et al., 2015)

$2.4 \mathrm{ACN}$ :

Demuestra atención condicional negativa

MMCOS (Smith et al., 2015) 2.5 CP: Utiliza un evidente control personal

MMCOS (Smith et al., 2015)
El monitor utiliza estrategias de afirmación del poder diseñadas para humillar y menospreciar a los participantes.

Bartholomew, K. J., Ntoumanis, N., y Thøgersen-Ntoumani, C. (2010). The controlling interpersonal style in a coaching context: Development and initial validation of a psychometric scale. Journal of Sport and Exercise Psychology, 32(2), 193-216.

El monitor omite atención y afecto cuando los atributos o comportamientos deseados no son mostrados por los participantes.

Assor, A., Roth, G., y Deci, E.L. (2004). The emotional costs of parents' conditional regard: A selfdetermination theory analysis. Journal of Personality, 72(1), 47-88.

El monitor ejerce un excesivo control personal y participa en comportamientos excesivamente intrusivos, como intentar interferir en aspectos de la vida de los participantes que no están directamente asociados con su realización de la actividad.

Fraser-Thomas, J., y Côté, J. (2009). Understanding adolescents' positive and negative developmental experiences in sport. The Sport Psychologist, 23(1), 3-23
1: El monitor/a emplea un gesto por la correcta realización de la tarea (por ejemplo, levanta el dedo pulgar)

2: El monitor/a da una respuesta verbal por la realización correcta de la tarea

3: El monitor/a ofrece una respuesta verbal acompañada de un gesto por la correcta realización de la tarea. (ej. Respuesta verbal y aplauso a la vez)

4: El monitor/a ofrece respuesta verbal y contacto por la correcta realización de la tarea (ej. Da respuesta verbal y toca el hombro del participante)

1: El monitor/a le dice que le gustaría que lo haga de cierta manera. "Esto me gustaría que lo hagas así"

3: El monitor/a le dice que quiere que algo lo haga de cierta manera "esto quiero que lo hagas asín"

5: El monitor/a le dice que debería hacer algo de cierta manera "esto deberías hacerlo así"

1: El monitor/a grita al participante, pero con un claro tono negativo, no es porque se encuentre lejos de este y necesite subir la voz para que lo oigan.

2: El monitor/a insulta al participante, por ejemplo "eres tonto", "eres un inútil" ...

3: El monitor/a intimida al participante con una amenaza, por ejemplo "ya verás ya...", "tú sigue así sí..."

4: El monitor/a intimida al participante con una amenaza que tendrá consecuencias, por ejemplo, "sigue, sigue que vas a volver a jugar..."

5: El monitor/a hace evidencia de algún intento de violencia o caso claro de ella, por ejemplo, simular dar un golpe o dar una "colleja"

1: El monitor/a omite la atención cuando el participante lo está haciendo mal.

2: El monitor/a omite la atención al participante justo tras realizar la acción incorrecta.

3: El monitor/a no le presta atención al participante tras realizar la acción incorrecta hasta la próxima actividad.

4: El monitor/a no le presta atención al participante tras realizar la acción incorrecta incluso hasta dos o más actividades posteriores (o por ejemplo la mitad de la clase). 5: El monitor/a no le vuelve a prestar atención al participante hasta el final de la clase.

1: El monitor/a pide a los participantes realizar trabajo extra sobre la actividad física dentro de su tiempo personal.

2: El monitor/a le hace 1 pregunta de tipo personal, que no hace referencia ni a la actividad que realiza o al interés por el estado de salud del propio participante.

3: El monitor/a le hace 2 preguntas de tipo personal, que no hace referencia ni a la actividad que realiza o al interés por el estado de salud del propio participante.

4: El monitor/a le hace 3 preguntas de tipo personal, que no hace referencia ni a la actividad que realiza o al interés por el estado de salud del propio participante.

5: El monitor/a le hace 4 o más preguntas de tipo personal, que no hace referencia ni a la actividad que realiza o al interés por el estado de salud del propio participante.

Podríamos considerarla de nivel superior si la pregunta afecta a un nivel de intimidad que no procede en absoluto 


\section{Análisis de la calidad del dato de un instrumento de observación del clima motivacional}

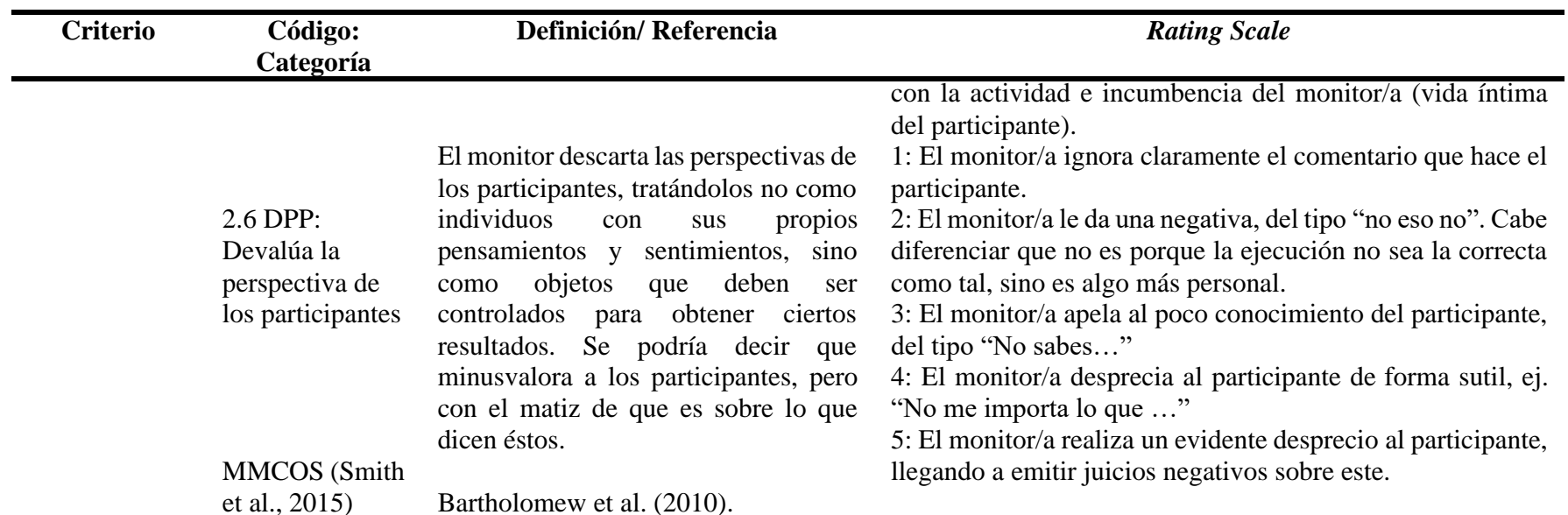

3. Implicado en la tarea
El entrenador enfatiza los criterios autorreferenciados como indicadores de éxito, como la mejora personal y el dominio de tareas, promoviendo así percepciones de competencia relacionadas con las tareas.

Duda, J.L., y Balaguer, I. (2007). Coach-created motivational climate. In S. Jowett y D. Lavallee (Eds.), Social psychology in sport. Champaign, IL: Human Kinetics.

\subsection{FCT:}

Enfatiza con

feedback la competencia de respuesta centrada en la tarea

MMCOS (Smith et al., 2015) 3.2 EIP: Explica la importancia del papel del participante

MMCOS (Smith et al., 2015)

\subsection{REM:}

Reconoce el esfuerzo y/o mejora

MMCOS (Smith et al., 2015)
El monitor comunica al participante mediante retroalimentación cuando realiza una respuesta que se centra en el buen hacer y/o aprender.

El monitor da información sobre la relevancia que tiene el participante dentro de la actividad que se realiza, acerca de cómo su figura es relevante en cuanto a actitud, competencia, o participación.

El monitor reconoce y hace saber al participante cuando éste se esfuerza en la tarea que realiza o aumenta el rendimiento o calidad del trabajo que lleva a cabo. Esto se enfatiza independientemente de que resultado sea positivo o negativo.
1: El monitor/a da Feedback verbal sobre una actividad. 3: El monitor/a da retroalimentación y además como seguir mejorando en la tarea que realiza.

5: El monitor/a da retroalimentación verbal, como seguir mejorando y además algún contacto (Ej. Tocar el brazo o el hombro)

1: El monitor/a explica la importancia de la competencia del propio participante con la tarea que va a realizar o realiza por ejemplo "es importante que tengáis ganas de hacer..."

2: El monitor/a da oportunidad e incita a mejorar o demostrar esa competencia al participante, por ejemplo "Venga, a ver como podéis mejorar eso"

3: El monitor/a explica la importancia de la actitud del participante respecto a la tarea a realizar. Por ejemplo, cabrían frases del tipo "es importante tener aquí mucha atención puesta".

4: El monitor/a da oportunidad e incita a mejorar o demostrar la actitud del participante.

5: El monitor/a explica la importancia de la participación en la tarea o la clase del propio participante por ejemplo "Vamos toda la participación es importante"

1: El monitor/a resalta una pequeña mejora o progreso. (Ej. "ya puedes levantar más el brazo en este ejercicio"

3: El monitor/a resalta que se está produciendo una mejora o progreso en el tiempo (Ej. "aún no estas al 100\% pero vas mejorando cada día")

el 5: El monitor/a resalta una mejora o progreso elevado (Ej. "te estas esforzando mucho") 


\section{Crespillo-Jurado, M., Anguera, M. T., Reigal, R. E., Hernández-Mendo, A.}

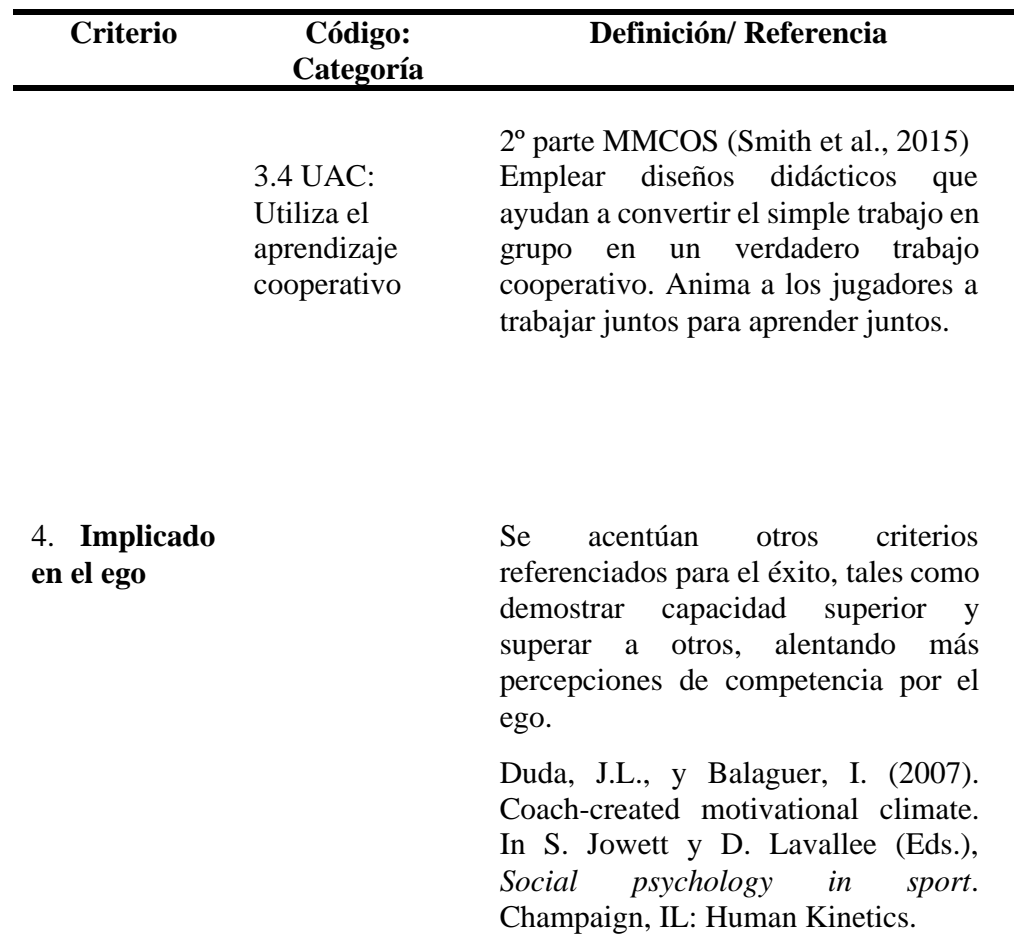

4.1 PE: Penaliza los errores

MMCOS (Smith et al., 2015)

4.2 RIS:

Enfatiza/recono ce

inferior/superior rendimiento y

habilidad

MMCOS (Smith et al., 2015)
1: El monitor/a propone una actividad cooperativa en el que no hay participación por parte de los participantes.

2: El monitor/a propone una actividad cooperativa en el que hay un $25 \%$ de participación.

3: El monitor/a propone una actividad cooperativa en el que hay un $50 \%$ de participación.

4: El monitor/a propone una actividad cooperativa en el que hay un $75 \%$ de participación.

5: El monitor/a propone una actividad cooperativa en el que hay un $100 \%$ de participación.

Seifriz, J.J., Duda, J.L., y Chi, L.K. (1992). The relationship of perceived motivational climate to intrinsic motivation and beliefs about success in basketball. Journal of Sport \& Exercise Psychology, 14(4), 375-391.

El monitor castiga o sanciona las acciones realizadas de forma incorrecta según la norma establecida para la correcta realización.

1: El monitor/a amonesta verbalmente al participante tras un error realizado por ejemplo " $\mathrm{O}$ ye, eso no!"

2: El monitor/a saca una "tarjeta amarilla" es decir podríamos considerarlo más "grave" aunque no tiene porqué ser como las de fútbol, habría una amonestación y además una advertencia por ejemplo "si vuelves a fallar, no juegas más a este juego".

3: El monitor/a saca una "tarjeta roja" no tiene porqué ser como en fútbol que signifique la expulsión del partido sino considerarlo más "grave" por ejemplo "como has fallado estas fuera ya"

4: El monitor/a expulsa fuera del juego a un participante tras cometer un error por ejemplo "iSal de la pista ya!" no hay advertencia previa ni razonamiento.

5: El monitor/a pone un castigo al participante tras cometer un error, puede ser del tipo: dar vueltas por el campo, realizar otro ejercicio, etc. Por ejemplo "como fallaste ayer en el juego hoy vas a dar dos vueltas más a la pista".

El monitor marca diferencias entre 1: El monitor/a le enfatiza que lo hace peor que otro, una $\begin{array}{ll}\text { rendimiento y/o habilidades en sus comparativa negativa. Por ejemplo, "lo haces peor que..." } \\ \text { niveles alto y bajo dentro de la tarea. } & \text { 2: El monitor/a hace una comparación positiva "lo haces }\end{array}$ mejor que..." enfatizando el superior.

3: El monitor/a enfatiza cierta igualdad que puede ser, "lo haces tan bien como..." o "lo haces tan mal como...".

4: El monitor/a enfatiza que "tiene que hacerlo mejor que..." por lo que también se reflejaría el inferior, en este caso no cabría mucho el otro polo el decir "tienes que hacerlo peor que..." 


\section{Análisis de la calidad del dato de un instrumento de observación del clima motivacional}

\begin{tabular}{|c|c|c|c|}
\hline Criterio & $\begin{array}{l}\text { Código: } \\
\text { Categoría }\end{array}$ & Definición/ Referencia & Rating Scale \\
\hline & & & $\begin{array}{l}\text { 5: El monitor/a enfatiza en superlativo que puede ser "eres } \\
\text { el que mejor lo hace" o podría pensarse como "eres el que } \\
\text { peor lo hace" }\end{array}$ \\
\hline & $\begin{array}{l}\text { 4.3 FR: } \\
\text { Fomenta la } \\
\text { rivalidad intra } \\
\text { equipo }\end{array}$ & $\begin{array}{l}\text { El monitor fomenta la rivalidad } \\
\text { haciendo hincapié en la importancia de } \\
\text { superar a los compañeros. Creando un } \\
\text { sentimiento de enfrentamiento entre } \\
\text { los participantes dentro del propio } \\
\text { grupo. }\end{array}$ & $\begin{array}{l}\text { 1: El monitor/a dice en clase que es importante ser superior } \\
\text { a los otros, se dirige a todo el grupo. } \\
\text { 3: El monitor/a en casos particulares dice cuanto falta para } \\
\text { superar a otros "te quedan } 5 \text { saltos para superar a X..." } \\
\text { 5: El monitor/a recalca sobre un caso concreto que ha } \\
\text { superado a otro "¡Bien, has superado a los demás!”. }\end{array}$ \\
\hline
\end{tabular}

\section{Apoyo a la relación}

5.1 API: Se asegura de que los participantes estén incluidos en las instrucciones/ac tividades/ejercic ios

MMCOS (Smith et al., 2015) 5.2 CNI:

Entabla/ permite conversaciones no instruccionales con los participantes

MMCOS (Smith et al., 2015)

5.3 IPP:

Muestra interés y preocupación por los participantes $1^{\circ}$ parte MMCOS (Smith et al., 2015)

El entorno fomenta sentimientos de cuidado, aceptación, inclusión, confianza y respeto, y esto se comunica de una manera cálida, positiva, consistente y de no contienda.

Fry, M.D., y Gano-Overway, L.A. (2010). Exploring the contribution of the caring climate to the youth sport experience. Journal of Applied Sport Psychology, 22(3), 294304.

Sarason, I.G., Sarason, B.R., y Pierce, G.R. (1990). Social support: The search for theory. Journal of Social and Clinical Psychology, 9(1), 133147.

El monitor comprueba y se asegura de que todos los participantes están formando parte de las instrucciones/actividades/ejercicios, y que nadie queda excluido de ellos.

El monitor habla con los participantes acerca de temas distintos a la actividad deportiva.

Permitiendo que estas conversaciones se lleven a cabo.

El monitor mira por los participantes y siente inquietud por lo que les ocurre. Se interesa activamente por el bienestar de estos.

$2^{\circ}$ parte MMCOS (Smith et al., 2015)
1: El monitor/a le repite la información de nuevo mencionando o refiriendo a una persona.

2: El monitor/a le repite la información de nuevo mencionando o refiriendo a dos personas.

3: El monitor/a le repite la información de nuevo mencionando o refiriendo a tres o más personas.

4: El monitor/a adapta un ejercicio para que puedan llevarlo a cabo. (Ej. "cómo no puedes levantar tanto el brazo tu usa mejor esto")

5: El monitor/a adapta una actividad para que todos puedan realizar la tarea. (Ej. "Vamos a poner distintas alturas para que todos podáis lanzar")

1: El monitor/a solo escucha a los participantes hablar sobre temas que no tienen que ver con la clase o actividad física que realizan.

2: El monitor/a hace algún gesto u onomatopeya, por ejemplo, "ajá", "hum" ...

3: El monitor/a responde con "sí" o "vale".

4: El monitor responde o interviene en la conversación con algún comentario.

5: El monitor/a interviene haciendo más preguntas sobre el tema.

1: El monitor/a pregunta por un participante que no se encuentra.

2: El monitor/a pregunta por “¿Cómo está la persona?” aquí podemos valorar tanto que el participante se encuentre presente como que no.

3: El monitor/a muestra preocupación por ese participante. Se puede preocupar por todos, por ejemplo "Tened cuidadito" 


\section{Crespillo-Jurado, M., Anguera, M. T., Reigal, R. E., Hernández-Mendo, A.}

\begin{tabular}{|c|c|c|}
\hline Criterio & $\begin{array}{l}\text { Código: } \\
\text { Categoría }\end{array}$ & Definición/ Referencia \\
\hline & $\begin{array}{l}\text { MMCOS (Smith } \\
\text { et al., 2015) }\end{array}$ & \\
\hline & $\begin{array}{l}5.4 \text { EPP: Está } \\
\text { pendiente del } \\
\text { participante }\end{array}$ & $\begin{array}{l}\text { El monitor presta atención a los } \\
\text { participantes, está pendiente de ellos. }\end{array}$ \\
\hline & $\begin{array}{l}\text { MMCOS (Smith } \\
\text { et al., 2015) }\end{array}$ & \\
\hline & 5.5 B: Bromea & $\begin{array}{l}\text { El monitor hace bromas (incluye la } \\
\text { realización de bromas amistosas, } \\
\text { tratando de divertir o entretener), se } \\
\text { encuentra a gusto y/o se ríe (se } \\
\text { incluyen respuestas positivas a } \\
\text { bromas). }\end{array}$ \\
\hline & $\begin{array}{l}\text { 5.6 PD: Pide } \\
\text { disculpas }\end{array}$ & $\begin{array}{l}\text { El monitor realiza la reparación que } \\
\text { puede preceder a una observación } \\
\text { hostil, como para neutralizar la } \\
\text { hostilidad antes del hecho, o puede } \\
\text { venir detrás de la hostilidad, de } \\
\text { cualquier tipo, como para deshacer y } \\
\text { reparar el daño. }\end{array}$ \\
\hline & $\begin{array}{l}5.7 \mathrm{DA}: \mathrm{Da} \\
\text { afecto }\end{array}$ & $\begin{array}{l}\text { El monitor realiza actos iniciales y } \\
\text { receptivos de solidaridad activa y } \\
\text { afecto, así como ofrece ayuda a los } \\
\text { demás (ya sea material, técnica, } \\
\text { social...) }\end{array}$ \\
\hline
\end{tabular}

6.

Frustración

de la relación
Se caracteriza por ser frío, crítico, y marcado por la aceptación que es contingente al/lo (s) comportamiento (s) deseable (s).

\section{-Smith et al. (2015)}

6.1 EP: Excluye participantes de ciertas instrucciones/ac tividades/ejercic ios

MMCOS (Smith et al., 2015)
El monitor activamente excluye a participantes de ciertas actividades, quita a participantes del grupo.

-MMCOS (Smith et al., 2015)
4: El monitor/a hace más preguntas sobre el estado de ese participante.

5: El monitor/a transmite "buenos deseos" para el participante, también puede asumirse el que se acerque directamente si cree que le ha pasado algo durante la actividad.

1: El monitor/a mira a los participantes.

3: El monitor/a hace un gesto a alguien relativo a la actividad que está realizando.

5: El monitor/a mira y además señala otro subcriterio, por lo que significa que está más pendiente para darse cuenta de sì ha realizado algo mal, por ejemplo.

1: El monitor/a responde a un comentario gracioso realizado en clase.

2: El monitor/a dice un comentario gracioso en clase.

3: El monitor/a bromea con algún participante, diciendo algo que refiera al participante directamente.

4: El monitor/a bromea con varios participantes, apelando a estos directamente.

5: El monitor/a se ríe abiertamente (carcajadas, risas...)

1: El monitor/a matiza algo previamente para que no se tome a mal por parte de los participantes (Ej. "Esto no lo digo para que lo toméis a mal, solo por mencionarlo")

3: El monitor/a se disculpa en general delante de todos (Ej. "Espero que no os molestara...")

5: El monitor/a pide disculpas a alguien personalmente. (Ej. Por separado le dice al participante que siente mucho X)

1: El monitor/a dice "Hola" a algún participante (o todos) cuando llegan.

2: El monitor/a dice "Hola" acompañada de otra fórmula como "buenas”, "buenos días”, “¿Qué tal?” (este último hay que diferenciarlo de que no se trata de un interés en sí por el participante sino como fórmula de cortesía).

3: El monitor/a hace algún gesto de afecto como puede ser dar un abrazo, pasar el brazo por los hombres, agarrar el brazo...

4: El monitor/a ofrece algún recurso al participante (por ejemplo, ofrecer un pañuelo, agua...)

5: El monitor/a ofrece ayuda al participante (apoyo emocional, por ejemplo)

1: El monitor/a excluye no se dirige o evita el contacto con un participante.

2: El monitor/a excluye de las explicaciones a algún participante (por ejemplo, "esto no va para ti" o similar).

3: El monitor excluye sin motivo a algún participante de un ejercicio en particular por ejemplo "este ejercicio no lo hagas tú"

4: El monitor/a excluye sin motivos a varios participantes de algún ejercicio por ejemplo "vosotros no lo haréis"

5: El monitor/a excluye sin motivo a algún participante de una actividad completa por ejemplo "tu espérate fuera hasta que ellos acaben" 


\section{Análisis de la calidad del dato de un instrumento de observación del clima motivacional}

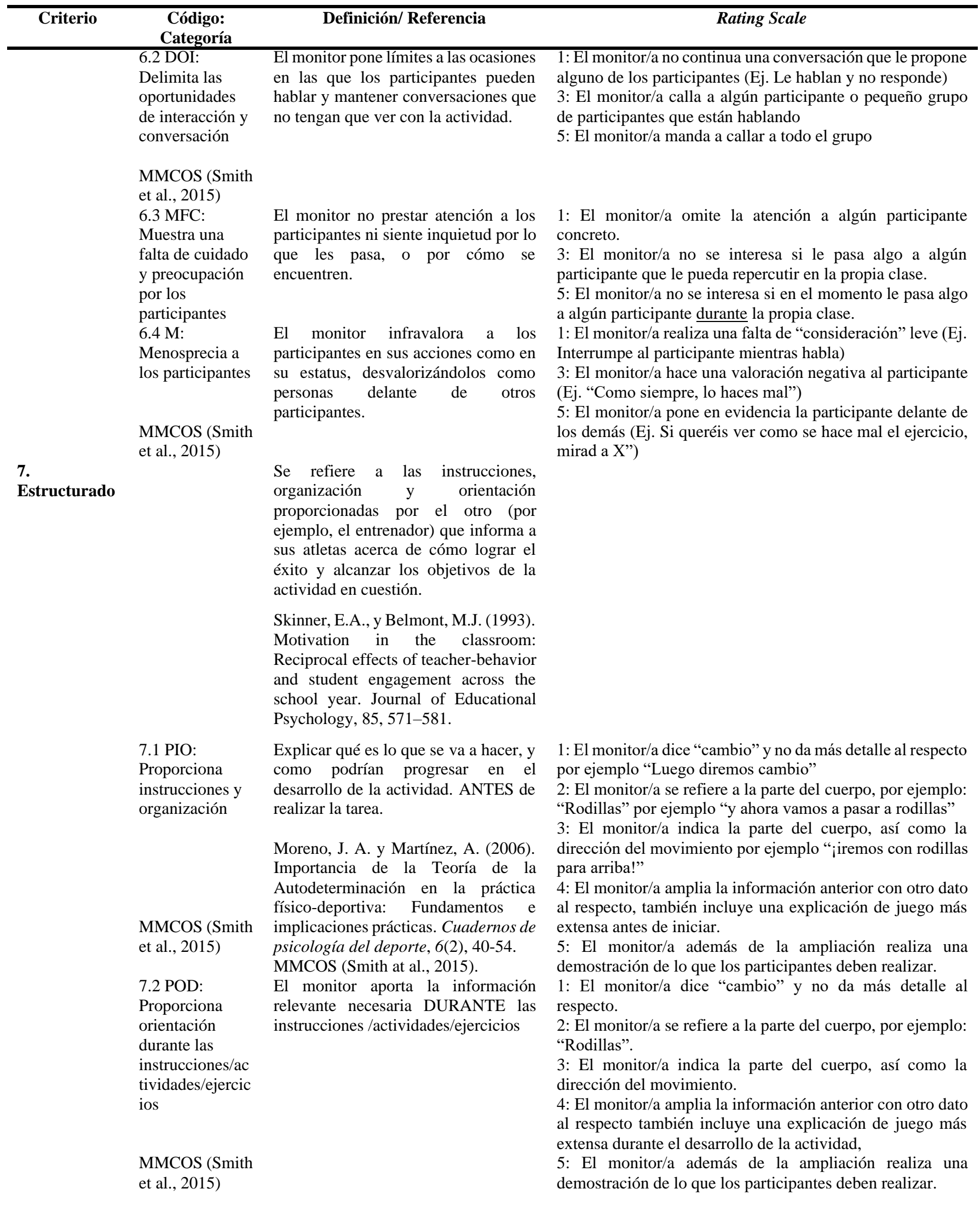




\section{Crespillo-Jurado, M., Anguera, M. T., Reigal, R. E., Hernández-Mendo, A.}

\begin{tabular}{|c|c|c|c|}
\hline Criterio & $\begin{array}{l}\text { Código: } \\
\text { Categoría }\end{array}$ & Definición/ Referencia & Rating Scale \\
\hline & $\begin{array}{l}\text { MMCOS (Smith } \\
\text { et al., 2015) } \\
7.4 \text { DS: Da una } \\
\text { sugerencia de } \\
\text { procedimiento o } \\
\text { de solución }\end{array}$ & $\begin{array}{l}\text { El monitor hace ver cómo incrementar } \\
\text { su competencia y sus habilidades. Así } \\
\text { conviene relacionar el contenido de la } \\
\text { instrucción, usando lenguaje y } \\
\text { ejemplos familiares, con sus } \\
\text { experiencias, sus conocimientos y sus } \\
\text { valores, Se puede además mostrar la } \\
\text { meta para la que puede ser relevante } \\
\text { aprender lo que se presenta. } \\
\text { Arana, J., Meilán, J., Gordillo, F., y } \\
\text { Carro, J. (2010). Estrategias } \\
\text { motivacionales y de aprendizaje para } \\
\text { fomentar el consumo responsable } \\
\text { desde la Escuela. REME, 13(35-36), } \\
\text { 19-39. } \\
\text { Incluir todos los actos que sugieren } \\
\text { maneras concretas de alcanzar una } \\
\text { meta deseada. }\end{array}$ & $\begin{array}{l}\text { 1: El monitor/a da una sugerencia para alcanzar una meta, } \\
\text { del tipo "Podrías hacerlo así..." } \\
\text { 2: El monitor/a al ver al participante realizarlo mal podría } \\
\text { decirle cómo hacerlo, del tipo "Esto hazlo así..." } \\
\text { 3: El monitor/a le presenta al participante una alternativa a } \\
\text { lo que está haciendo para conseguir el fin perseguido. } \\
\text { 4: El monitor/a le presenta una explicación detallada de la } \\
\text { realización de esa solución o alternativa propuesta al } \\
\text { ejercicio, encontramos la diferencia respecto a dar } \\
\text { instrucciones por ejemplo en cuanto a la especificidad de la } \\
\text { respuesta. } \\
\text { 5: El monitor/a le suma una demostración de la acción a } \\
\text { realizar. En este caso la diferencia la encontramos con el } \\
\text { apartado de da instrucciones, como en el caso anterior en } \\
\text { cuanto a la especificidad de la respuesta que se está dando. }\end{array}$ \\
\hline $\begin{array}{l}\text { 8. Silencio } \\
9 . \\
\text { Aprovechami } \\
\text { ento del } \\
\text { espacio }\end{array}$ & $\begin{array}{l}8.1 \text { Silencio } \\
9.1 \\
\text { Aprovechamient } \\
\text { o del espacio }\end{array}$ & $\begin{array}{l}\text { El monitor no habla. } \\
\text { Como organiza al grupo el monitor. }\end{array}$ & $\begin{array}{l}\text { No hay rating scale simplemente es SI } \\
\text { No hay rating scale } \\
\text { JG: Junta a todo el grupo } \\
\text { SI: Separa individualmente } \\
\text { PP: Pone en parejas } \\
\text { PG: Pone en grupos }\end{array}$ \\
\hline $\begin{array}{l}10 . \\
\text { Proxémica }\end{array}$ & 10.1 Proxémica & $\begin{array}{l}\text { Espacio entre el monitor/a y la gran } \\
\text { mayoría de los participantes o con los } \\
\text { que realiza la interacción concreta. }\end{array}$ & $\begin{array}{l}\text { No hay rating scale } \\
\text { DI: Distancia íntima (hasta } 45 \mathrm{~cm} \text {.) } \\
\text { DP: Distancia personal }(46-122 \mathrm{~cm} \text {.) } \\
\text { DS: Distancia social }(122-365 \mathrm{~cm} \text {.) } \\
\text { DPu: Distancia pública }(>365 \mathrm{~cm} \text {.) }\end{array}$ \\
\hline
\end{tabular}

